\title{
A QUANTUM MECHANICS AND MOLECULAR MECHANICS STUDY OF THE EFFECTS OF DIFFERENT SOLVENTS AND TEMPERATURES ON THE CONNECTIONS OF METHOTREXATE DERIVATIVES ANTICANCER DRUG TO NANOTUBES CARRIERS
}

\author{
V. KHODADADI ${ }^{a}$, N. HASANZADEH ${ }^{a^{*}}$, H. YAHYAEI $^{b}$ AND A. RAYATZADEH ${ }^{a}$ \\ ${ }^{a}$ Department of Chemistry, Ahvaz,Branch, Islamic Azad University, Ahvaz, Iran. \\ ${ }^{b}$ Department of Chemistry, Zanjan Branch, Islamic Azad University, Zanjan, Iran.
}

\begin{abstract}
In this study, the interactions of two effective derivatives of the methotrexate anticancer drug with Single-wall carbon nanotubes (SWNTs) and Boron nitride nanotubes (BNNTs) in the gas phase were investigated using the DFT calculations. Through the DFT method, the effects of different solvents on the interaction of methotrexate derivatives with SWNTs and BNNTs within the Onsager self-consistent reaction field (SCRF) model, as well as the effects of temperature on the stability of interactions between compounds in various solvents were studied. Thermodynamic parameters, Frontier Molecular Orbitals (FMOs) and Total Density of States (DOS) of the title compounds were also studied using theoretical calculations. Molecular properties of the structures such as the ionization potential (I), electron affinity (A), chemical hardness $(\eta)$, electronic chemical potential $(\mu)$ and electrophilicity $(\omega)$ were investigated as well. SWNTs are more suitable carriers for L-MTX and BNNTs are more suitable carriers for L-FMTX. Also, the interaction of methotrexate derivatives with SWNTs and BNNTs was examined via Amber, Opls, Charmm and MM+ force fields through the molecular mechanic (MM) method. The calculations were carried out through the Monte Carlo simulation methods at different temperatures. The effects of gas phase and various solvent media with different dielectric constants (water, DMSO, methanol, ethanol, $\mathrm{CH} 2 \mathrm{Cl} 2$ and DMF) on the interaction of methotrexate derivatives were investigated using the aforementioned force fields. The MM+ force field, which is an exclusive force field for calculations related to macromolecules, had the lowest amount of energy and featured the most stable form of connection for Methotrexate derivatives connected to SWNTs and BNNTs. The most significant finding is that with respect to both thermodynamic properties and conformer populations, the Monte Carlo and Molecular Mechanics-Quantum Mechanics results are in agreement.
\end{abstract}

Keywords: Quantum Monte Carlo (QMC), Single-wall carbon nanotubes (SWNTs), Boron nitride nanotubes (BNNTs), methotrexate derivatives anticancer drug, Solvent effect, force field.

\section{INTRODUCTION}

Carbon nanotubes (CNTs) possess extraordinary properties and are unique nano systems. In such nanotubes, carbon atoms are interconnected through covalent bonds. These nanotubes include Single-walled nanotubes (SWNTs) and Multi-walled nanotubes (MWNTs) [1-3]. SWNTs are considered as one of the most suitable items for being used in biological systems due to their appropriate size, biocompatibility, controllable properties and ability to have reversible responses compared to biochemicals. For instance, their small size allows them to easily pass through shells and biological barriers and enter cells; they have a diameter about half of that of a DNA strand [4-5]. The applications of nanotubes and their use as drug nano-carriers have received a lot of attention recently [6-7]. In particular, functional drug-containing nanotubes (drug nano-carriers) have helped develop a new generation of drugs and have opened a new chapter of treatment in medical science [8-9]. Previous studies have proved that carbon nanotubes are not inherently toxic. Therefore, these nanotubes can be suitable options for being used as carrier nanotubes and in drug delivery [10-11].

The Boron nitride nanotube (BNNT) is a Boron compound. It is a white solid with a layered structure that resembles Graphite. In the boron-nitride structure, the strong B-N ionic bond and the covalent bond are formed simultaneously along two adjacent boron nitride nanotube layers, creating the folded single structure [12]. Nanostructures, especially boron nitride (BN) nanostructures, have great potentials for applications in pharmaceutics, medicine and industry [13-15]. BNNTs possess high elastic and tensile strength and high resistance to oxidation in air at high temperatures; they also withstand deformations more efficiently [16-17]. In Addition, BNNTs have been found to be good insulators with a large band gap $(\sim 5.5 \mathrm{eV})[18]$. On the other hand, the thermal conductivity of BNNTs is comparable to that of CNTs, though they have better thermal stability [19]. Thus, the mechanical stiffness of BNNTs is assumed to be comparable to that of CNTs as well [20]. Furthermore, boron nitride nanotubes are non-toxic in nature compared to CNT, which makes them suitable for possible biomedical applications [21]. To minimize the toxic effects of drugs, especially anti-neoplastic drugs, drug delivery systems are used to improve cancer cell specificity of a drug during chemotherapy [22-23]. In the case of methotrexate, BNNTs are used to make the drug more water-soluble, thereby making the transportation of MTX via the bloodstream easier. Thus, there is a theory that a more water-soluble MTX-BNNT has a greater therapeutic activity [24-25]. Analysis of electrical properties including state density, charge distribution and charge transfer in the case of BNNTs containing drug molecules shows that there are changes in the molecular properties of the drug. In fact, this study attempts to help reveal more information about the experimental stages of these drugs and their interaction with BNNTs, so that they can be used experimentally.

Methotrexate (MTX) has become the most widely used second-line agent for the treatment of cancerous cells [26]. To date, MTX has been used to treat patients who have rheumatoid arthritis (RA) or other inflammatory diseases; it has also been employed to treat a number of cancers [27] and some other autoimmune diseases [28]. MTX is a folic acid analogue used as a reproduction agent. As folate receptors are over-expressed on the cell membranes of many types of cancer cells, MTX is an effective treatment for cancers [29]. Figure 1 [30] shows the working mechanism of MTX.

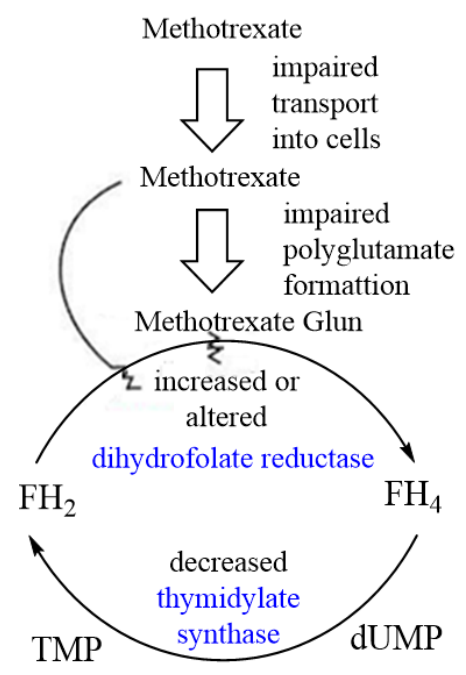

Figure 1. The mechanism of MTX drug [30].

The structure of MTX with the formula $\mathrm{C} 20 \mathrm{H} 22 \mathrm{~N} 8 \mathrm{O} 5$ can be seen in Figure 2. MTX is a competitive inhibitor of folic acid production [31]. Various modifications in the glutamic acid moiety have been reported with the aim of decreasing the formation rate of poly- $\gamma$-glutamates in normal cells; they include: substitution of $\gamma$-carboxyl with amide or peptide groups and replacement of glutamic acid with other amino acids; these steps have been taken with the goal 
of generating a new class of antitumor agents. We prepared several MTX derivatives containing R- or $\gamma$-substituted glutamic acids [32]. The lysine and ornithine derivatives were synthesized from MTX, and both derivatives had selfsame bindings [33].

Various derivatives of 2,4-diamino, N10-methylpteroyl glutamic acid (MTX) have been synthesized by modifying $\gamma-\mathrm{COOH}$, such as MTX-N (idoacetyl) Llysine, $\mathrm{Cbz}$ and $\mathrm{NH} 2$ groups. Moreover, some of the derivatives of MTX - such as Lysyl derivatives - have been synthesized through the reaction of $\gamma-\mathrm{COOH}$ with the amine group of lysine. These derivatives vary with respect to the number of Lysyl groups attached to them. Thus, all other derivatives exhibit less activity. The attachment of some functional groups causes changes in MTX, leading to the formation of a number of MTX derivatives [34-35]. Also, halogenated derivatives of methotrexate have been evaluated as inhibitors of human dihydrofolate reductase in cancer chemotherapy [36]. Given the wealth of attention they have received in numerous studies and their medicinal properties, the following two derivatives were used in this article (Fig 2):<smiles>[X]C(C[C@H](NC(=O)c1ccc(N(C)Cc2cnc3nc(N)nc(N)c3n2)cc1)C(=O)O)C(=O)O</smiles>

Figure 2. Chemical structure of MTX derivatives: L-MTX (1): X=H (4-(((2, 4-diaminopteridin-6-yl) methyl) (methyl) amino) benzoyl)- $L$-glutamic acid and L-FMTX (2): X=F (2S)-2- (4-(((2, 4-diaminopteridin-6-yl) methyl) (methyl) amino) benzamid)-4 -fluoropentanedioic acid.

Nowadays, designing and simulating medicine with the help of computers and specialized software have become particularly important [37]. Through this method, it is possible to save time and costs in developing new drugs by identifying the drug molecule and the receptor in the body and by using techniques that evaluate the interaction of these compounds in the same environment [38-39]. The use of computational methods plays an important role in understanding and optimization of laboratory processes that aim to evaluate the drug delivery capability of drug carriers. Computational simulation, which employs computational chemistry software used in pre-laboratory research to produce more effective drugs with less side effects, can lead to faster and more cost-effective prognosis, diagnosis and treatment in cancer patients [40-41].

In this study, structural, thermo-dynamic and electronic information for SWNTs and BNNTs with MTX derivative complexes have been presented using quantum and Monte Carlo calculations as well as molecular mechanics over a range of temperatures and solvents [42-43]. Thus, by comparing the energies computed through Monte Carlo calculations in the CHARMM, AMBER, MM+ and OPLS force fields, the differences in the complexes resulting from the incorporation of the MTX derivatives into SWNTs and BNNTs are demonstrated [44-45]. In addition to investigating the effects of interactions of MTX derivatives with SWNTs, the interactions in the gas phase as well as in the solvents DMF, DMSO, water, ethanol and methanol were also studied using different force fields and Monte Carlo calculations; the same procedure was followed for the interaction between MTX derivatives and BNNTs. The present study will help gain deeper insights about the experimental stages of these drugs and their interaction with SWNTs and BNNTs, so that they can be used experimentally.

\section{Computational Method}

In this study, the quantum chemical calculations were performed using the Gaussian 09W software [46]. The molecular structure of the title compounds in the ground state was optimized using the Density Functional Theory (DFT/B3LYP/6-31+G*) [47]. The Polarized Continuum Model (PCM) [48][47], The Frontier Molecular Orbital (FMO) analysis and electronic properties such as energies of HOMO and LUMO orbitals, HOMO-LUMO energy gap (Eg), ionization potential (I), electron affinity (A), global hardness ( $\eta$ ), electronegativity $(\chi)$, electronic chemical potential $(\mu)$, electrophilicity $(\omega)$, and chemical softness (S) were estimated through the EHOMO and ELUMO energies using the B3LYP.6-31+G* level of theory [49-52].
The optimized molecular structures, Molecular Electrostatic Potential (MEP) maps and UV.Vis spectra were visualized using GaussView 05 program [47] There are three types of QMC: variation, diffusion and Green's functions. These methods act with an openly correlated wave function and calculate integrals numerically, utilizing a Monte Carlo integration. These calculations are very time consuming, but they are the most accurate methods known to date. Overall, DFT calculations provide perfect and increasingly more accurate quantitative results as the molecules under consideration become smaller [53]. DFT methods are accessible in macro model programs as well. It is vital to select a level that is well-parameterized for the molecular system being investigated. Conformational interconversions are governed by precise energy parameters and geometry coordinates, which are vital in molecular systems, too. We chose the Low-energy structures found on each surface and subjected them to unrestrained quantum mechanical minimization Using B3LYP.6-31+G* SCRF [54].

Moreover, the calculations related to the interactions between MTX and Single wall carbon nanotubes (SWNTs) and Boron nitride nanotube (BNNTs) have been carried out using each of the force fields AMBER, OPLS, CHARMM and MM+ (Fig. 3 and Fig. 4). MTX derivatives are placed inside the carbon and boronnitride nanotubes. This method is utilized in HyperChem software. Four different force fields are available in the Macro Model program. Choosing a force field that is well parameterized for the molecular system under study is very important [55-56].

The Monte Carlo method is one of the most broadly and commonly used numerical techniques, with applications in statistical physics, quantum mechanics, field theory etc [57]. With its ability to generate a canonical ensemble, Monte Carlo simulation is applied when systems have difficult integrals to be solved and should generate some random numbers to yield statistically fixed and independent values [58-59]. Due to its simplicity, a metropolis algorithm is applied more frequently than other algorithms in the Monte Carlo method [60]. Random displacement is used to determine the accuracy of the algorithm. Every move can be accepted in minor displacements; however, only a few moves are acceptable in large cases. In this study, differences in force fields are illustrated by comparing the energies calculated using force fields AMBER, OPLS, CHARMM and MM+. HyperChem professional release 7.01 is used for the Molecular Mechanics calculations. Geometry optimization as well as Monte Carlo simulation were performed using this software [11].
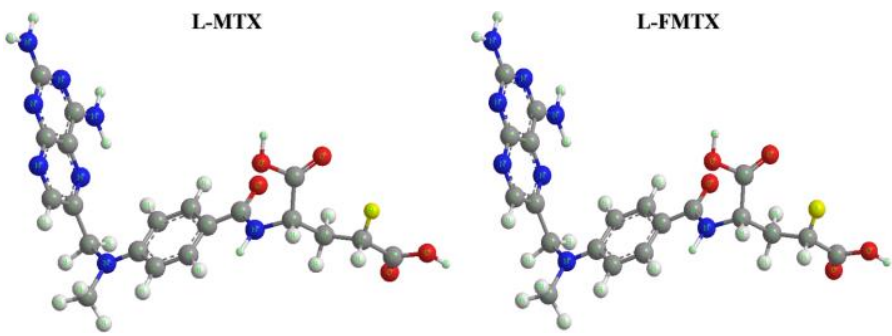

Figure 3. The theoretical geometric structure of the L-MTX and L-FMTX (optimized by B3LYP.6-31+G level).

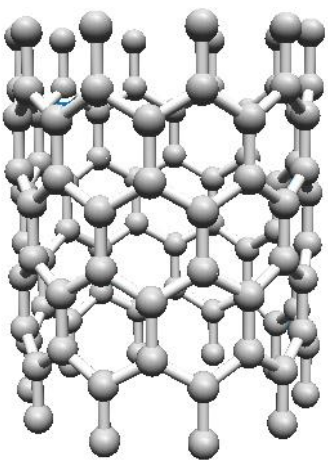

(SWNTs)

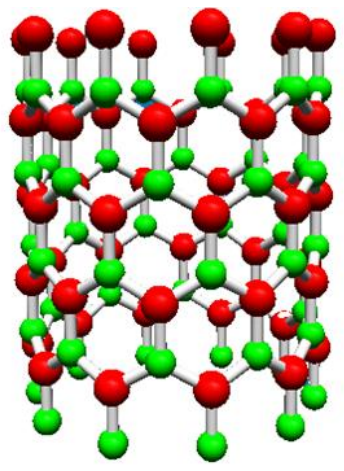

(BNNTs)
Figure 4. Single-wall carbon nanotubes (SWNTs) and Boron nitride nanotube (BNNTs) (optimized by B3LYP.6-31+G level). 


\section{RESULTS AND DISCUSSION}

In macromolecules, thermodynamic parameters such as enthalpies, entropies, and free energies depend on many conformational degrees of freedom that these flexible molecules can take. The free energies of macromolecules in solutions cannot typically be estimated using Monte Carlo simulations, partially because transitions from one conformer to another occur infrequently. In addition, Molecular Mechanics simulations regularly succeed in providing more efficient samplings of conformational space in the case of macromolecules. What Monte Carlo or Molecular Dynamics simulations can achieve, however, is estimating free energy differences between similar systems. Such calculations allow, for example, comparison of binding affinities of similar drug molecules with the target receptor, thus facilitating rational design of more potent and selective drugs.

A word of caution is due here, however. Monte Carlo sampling of harmonic potentials yields classical probability distributions, while bond vibrations in the real MTX atoms with carbon nanotube molecules are quantized. Consequently, classical Monte Carlo simulations fail to precisely reproduce such thermodynamic properties as heat capacity or vibrational entropy of isolated molecules. Therefore, in this section we have used the quantum mechanics methods.

Quantum chemical methods are highly useful tools for acquiring information about molecular structures and electrochemical behaviors. A Frontier Molecular Orbitals (FMO) analysis was carried out for the compounds using the B3LYP/6$311+\mathrm{G}(\mathrm{d})$ level [61]. FMO results such as EHOMO, ELUMO and the HOMOLUMO energy gap $(\Delta \mathrm{E})$ of the title compounds have been summarized in Table 1. Table 9 presents important information regarding quantum mechanics calculations and the stability of MTX derivatives with carbon and boron-nitrite nanotubes. In this step, the L-MTX molecule was first optimized alone and then with carbon and boron-nitrite nanotubes.

The energy level of the LUMO and HOMO and their energy gaps reflect the chemical reactivity of the molecule [62]. In addition, the HOMO can act as an electron donor and the LUMO as an electron acceptor. An increased level of HOMO energy (EHOMO) for the molecule leads to a heightened ability to donate electrons to a suitable acceptor molecule that has a low-energy empty molecular orbital. $\mathrm{E}_{\text {Hомо }}$ and $\mathrm{E}_{\mathrm{LUMO}}$ are related to ionization potential $\left(I=-E_{\text {Номо }}\right)$ and electron affinity $\left(A=-E_{L U M O}\right)$, respectively [63-65]. Global hardness $(\eta)$, electronegativity $(\chi)$, electronic chemical potential $(\mu)$, electrophilicity $(\omega)$ and chemical softness $(S)$ parameters [66] are calculated using the following equations:

$$
\begin{aligned}
& (\eta=I-A / 2) \\
& (\chi=I+A / 2) \\
& (\mu=-(I+A) / 2) \\
& \left(\omega=\mu^{2} / 2 \eta\right) \\
& (S=1 / 2 \eta)
\end{aligned}
$$

The values of these parameters are reported in Table 9. The global hardness $(\eta)$ parameter is related to the energy gap $\left(\mathrm{Eg}=\mathrm{E}_{\mathrm{LUMO}}-\mathrm{E}_{\mathrm{HOMO}}\right)$ and defined as the charge transfer resistance of an atom or a group of atoms.

As shown in Table 1, the HOMO energy of the compound L-MTX with SWNTs has the lowest value $(-0.29841 \mathrm{eV})$. A large energy gap is indicative of high stability for the molecule. The HOMO-LUMO energy gap $(\Delta \mathrm{E})$ values calculated for the structures L-MTX with SWNTs and L-MTX with BNNTs are 0.15774 and $0.02094 \mathrm{eV}$, respectively. The results show that L-MTX with SWNTs is more stable. DOS plots also demonstrate the energy gaps $(\Delta \mathrm{E})$ calculated for the L-MTX (see Fig. 5). Table 9 shows the specifics of quantum molecular descriptors of title compounds such as electron affinity, ionization potential, electronic chemical potential, global hardness and electrophilicity. The chemical hardness $(\eta)$ values for the compounds L-MTX with SWNTs and LMTX with BNNTs are $0.07887 \mathrm{eV}$ and $0.01047 \mathrm{eV}$, respectively. The L-MTX with SWNTs has the highest chemical hardness $(\eta=0.07887 \mathrm{eV})$; therefore, it is a hard, less reactive molecule with a wide energy gap $(\Delta \mathrm{E}=0.15774 \mathrm{eV})$.

As a form of potential energy, electronic chemical potential $(\mu=-(I+A) / 2)$ has the ability to be absorbed or released during chemical reactions and might also be modified during phase transitions. The electronic chemical potential of Methotrexate with DWNTs has the largest negative value $(-0.012572 \mathrm{eV})$.
Electrophilicity $(\omega)$ is a measure of energy stabilization for when the system receives an additional electronic charge from the environment. This index $(\omega=\mu 2 / 2 \eta)$ holds information about both electron transfer (chemical potential) and stability (hardness); it also describes global chemical reactivity more precisely. The higher the value of electrophilicity index, the higher the capacity of the molecule to accept electrons. The electrophilicity index for the L-MTX with SWNTs and L-MTX with BNNTs are 0.305552 and $2.719402 \mathrm{eV}$, respectively. The L-MTX with SWNTs has the highest electrophilicity index; therefore its capacity for accepting electrons is quite high.

The dipole moment $(\mu \mathrm{D})$ is an appropriate measure of the asymmetric nature of molecules. The composition and dimensionality of the 3D structures determine its magnitude. As shown in Table 9, all structures have a high value of dipole moment and a point group of $\mathrm{C} 1$, which indicates the lack of symmetry in the structures. The dipole moment for the L-MTX with SWNTs (B3LYP/6$31+\mathrm{G}(\mathrm{d})=2.8907$ Debye $)$ is lower than that for L-MTX with BNNTs $(9.7738$ Debye, respectively). The asymmetric character of L-MTX with BNNTs is the reason behind its high dipole moment value.

As can be seen in Table 9, the HOMO energy of the compound L-FMTX with BNNTs has the lowest value $(-0.29522 \mathrm{eV})$. A large energy gap points to a high level of stability for the molecule. The HOMO-LUMO energy gap $(\Delta \mathrm{E})$ values calculated for the structures L-FMTX with SWNTs and L-MTX with BNNTs are 0.04198 and $0.15458 \mathrm{eV}$, respectively. The results show that the compound $\mathrm{L}$ FMTX with BNNTs is more stable. DOS plots [43] also demonstrate the energy gaps $(\triangle \mathrm{E})$ calculated for the L-FMTX (Fig. 5).

Table 9 also shows the specifics of quantum molecular descriptors of title compounds, such as electron affinity, ionization potential, electronic chemical potential, global hardness and electrophilicity.

The chemical hardness $(\eta)$ values for the compounds L-FMTX with SWNTs and L-FMTX with BNNTs are $0.02099 \mathrm{eV}$ and $0.07729 \mathrm{eV}$, respectively. The LFMTX with BNNTs has the highest chemical hardness $(\eta=0.07729 \mathrm{eV})$; therefore, it is a hard, less reactive molecule with a wide energy gap $(\Delta \mathrm{E}=$ $0.15458 \mathrm{eV}$ ).

The electrophilicity index for the L-FMTX with SWNTs and L-FMTX with BNNTs is 1.575917 and $0.307242 \mathrm{eV}$, respectively. The L-FMTX with BNNTs has the highest electrophilicity index; therefore its capacity for accepting electrons is quite high. The dipole moment for the L-FMTX with SWNTs (B3LYP/6-31+G (d) =3.5689 Debye) is lower than that for the L-FMTX with BNNTs (2.6501 Debye, respectively).

As the data suggest, the amount of Gibbs free energy for L-MTX with a carbon nanotube and L-MTX with a boron-nitride nanotube is -9019.34004 Hartree and -5588.257224 Hartree, respectively. The data also indicate that the L-MTX and carbon nanotube combination has more stability. This is further confirmed by the amounts of HOMO and LUMO energies. However, the results pertaining to LFMTX differ substantially with those related to L-MTX. The amount of Gibbs free energy for L-FMTX with a carbon nanotube and L-FMTX with a boronnitride nanotube is -4966.985640 Hartree and -8397.302476 Hartree, respectively. The data point to the fact that the L-FMTX and boron-nitride nanotube combination has more stability, which is again confirmed by the amounts of HOMO and LUMO energies. Figure 4 shows the structures of LMTX and L-FMTX. The two structures differ in the $\mathrm{H}$ and $\mathrm{F}$ constituents. The stability of the L-MTX and carbon nanotube combination, as well as that of the L-FMTX and boron-nitride nanotube combination is due to high electronegativity of $\mathrm{F}$ compared to $\mathrm{H}$.

The DFT calculations have taken place in a gas phase [67-69]. The total energy of a molecule consists of the sum of translational, rotational, vibrational and electronic energies. The statistical thermochemical analysis of title compounds is carried out by placing the molecule at the room temperature of $25^{\circ} \mathrm{C}$ and under 1 atmospheric pressure. The thermodynamic parameters such as zero point vibrational energy, rotational constant, heat capacity (C) and entropy (S) of the title compound using the B3LYP/6-31+G (d) level are displayed in Table 1. According to this table, the values calculated for L-MTX with SWNTs are smaller than those for L-MTX with BNNTs. The results suggest that the compound L-MTX with SWNTs is more stable. However, in the case of LFMTX, the results are different. All the thermodynamic parameters, the enthalpy, the zero-point energy and the entropy are totally in line with the changes in Gibbs free energy. According to this table, the values calculated for L-FMTX with BNNTs are smaller than those for L-FMTX with SWNTs. The results suggest that the former compound is more stable. 
J. Chil. Chem. Soc., 66, N 4 (2021)

Table 1. The electronic properties of the Methotrexate calculated using the B3LYP/6-31+G* level of theory.

\begin{tabular}{|c|c|c|c|c|c|c|}
\hline Property & L-MTX & $\begin{array}{c}\text { L-MTX+ (C } \\
\text { nanotubes) }\end{array}$ & $\begin{array}{c}\text { L-MTX+ (BN } \\
\text { nanotubes) }\end{array}$ & L-FMTX & $\begin{array}{c}\text { L-FMTX+ (C } \\
\text { nanotubes) }\end{array}$ & $\begin{array}{c}\text { L-FMTX+ }(\mathrm{BN} \\
\text { nanotubes })\end{array}$ \\
\hline HF (Hartree) & -1569.0315828 & -9020.7871662 & -5589.3955307 & -1666.79514 & -4968.0516805 & -8398.9072602 \\
\hline Zero-point correction(Hartree) & 0.448227 & 1.570951 & 1.077289 & 0.436996 & 1.009014 & 1.491755 \\
\hline Thermal correction to Energy(Hartree) & 0.478087 & 1.686926 & 1.137363 & 0.468411 & 1.065096 & 1.603840 \\
\hline Thermal correction to Enthalpy(Hartree) & 0.479031 & 1.687870 & 1.138307 & 0.469355 & 1.066040 & 1.604784 \\
\hline Thermal correction to Gibbs Free Energy(Hartree) & 0.383664 & 1.447122 & 1.004015 & 0.368044 & 0.937894 & 1.366664 \\
\hline Sum of electronic and zero-point Energies(Hartree) & -1568.583356 & -9019.216216 & -5588.318242 & -1666.358144 & -4967.042667 & -8397.415506 \\
\hline Sum of electronic and thermal Energies(Hartree) & -1568.553496 & -9019.100241 & -5588.258168 & -1666.326729 & -4966.986585 & -8397.303420 \\
\hline Sum of electronic and thermal Enthalpies(Hartree) & -1568.552552 & -9019.099296 & -5588.257224 & -1666.325785 & -4966.985640 & -8397.302476 \\
\hline $\begin{array}{l}\text { Sum of electronic and thermal Free } \\
\text { Energies(Hartree) }\end{array}$ & -1568.647919 & -9019.340044 & -5588.391516 & -1666.427096 & -4967.113787 & -8397.540596 \\
\hline E (Thermal) (KCal.Mol) & 300.004 & 1058.562 & 713.706 & 293.932 & 668.358 & 1006.425 \\
\hline CV (Cal.Mol-Kelvi) & 113.735 & 528.853 & 296.206 & 117.599 & 274.149 & 506.952 \\
\hline S (Cal.Mol-Kelvin) & 200.717 & 506.696 & 282.642 & 213.228 & 269.706 & 501.165 \\
\hline Dipole moment (Debye) & 10.2493 & 2.8907 & 9.7738 & 3.2280 & 0.5689 & 2.6501 \\
\hline Point Group & $\mathrm{C} 1$ & $\mathrm{C} 1$ & $\mathrm{C} 1$ & $\mathrm{C} 1$ & $\mathrm{C} 1$ & $\mathrm{C} 1$ \\
\hline $\mathrm{E}_{\text {Номо }}(\mathrm{eV})$ & -0.28207 & -0.29841 & -0.24910 & -0.28577 & -0.27820 & -0.29522 \\
\hline $\mathrm{E}_{\text {LUMO }}(\mathrm{eV})$ & -0.19143 & -0.14067 & -0.22816 & -0.19260 & -0.23622 & -0.14064 \\
\hline $\mathrm{Eg}(\mathrm{eV})$ & 0.09064 & 0.15774 & 0.02094 & 0.09317 & 0.04198 & 0.15458 \\
\hline $\mathrm{I}(\mathrm{eV})$ & 0.28207 & 0.29841 & 0.24910 & 0.28577 & 0.27820 & 0.29522 \\
\hline $\mathrm{A}(\mathrm{eV})$ & 0.19143 & 0.14067 & 0.22816 & 0.1926 & 0.23622 & 0.14064 \\
\hline$\chi(\mathrm{eV})$ & 0.23675 & 0.21954 & 0.23863 & 0.239185 & 0.25721 & 0.21793 \\
\hline$\eta(\mathrm{eV})$ & 0.04532 & 0.07887 & 0.01047 & 0.046585 & 0.02099 & 0.07729 \\
\hline$\mu(\mathrm{eV})$ & 5.3121 & 2.8907 & 9.7738 & 1.9530 & 3.5689 & 2.6501 \\
\hline$\omega(\mathrm{eV})$ & 0.618387 & 0.305552 & 2.719402 & 0.614033 & 1.575917 & 0.307242 \\
\hline $\mathrm{S}(\mathrm{eV})$ & 11.03266 & 6.339546 & 47.75549 & 10.73307 & 23.82087 & 6.469142 \\
\hline
\end{tabular}



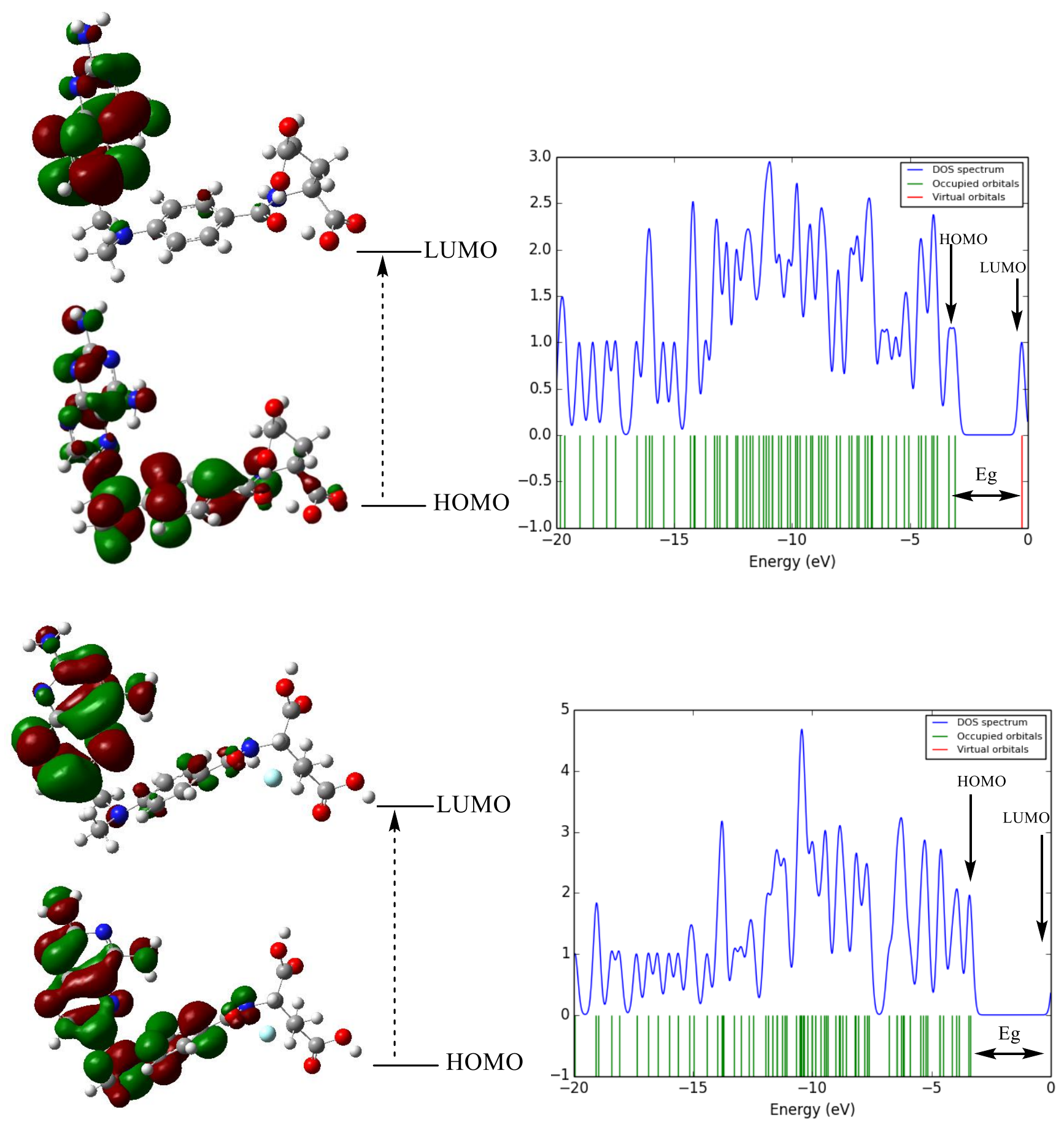

Figure 5. Calculated Frontier molecular orbitals and DOS plots of L-MTX and L-FMTX ( $\triangle$ E: Energy gap between LUMO and HOMO)

In the present study, calculations related to the interaction between MTX derivatives (L-MTX and L-FMTX) and Single Wall carbon nanotubes and Boron nitride nanotubes (SWNTs \& BNNTs) have been carried out using AMBER, OPLS, CHARMM (BIO+) and MM+ force fields. Biomolecules are complex systems. Their structures are represented by multidimensional rugged energy landscapes with a huge number of local minima separated by high energy barriers. Thus, any simulation study primarily deals with adequate description of the atomistic interaction or force field and convergence of the configuration space sampling of such a complex energy landscape. Efficient sampling can be achieved through enhanced conformational search techniques. The experimental values of the properties predicted by a force field are signs of its quality. There are four predominantly used force field families for molecular mechanic simulations at the time, including AMBER, OPLS, CHARMM (BIO+) and $\mathrm{MM}+$. These classic force fields have constantly been improved and verified; however, given the intricacies of the energy landscape, the successful application of these fields in many systems remains to be validated. Thus, how the employed force field affects the simulation results is a question worth investigating.

Among other appropriate tools for evaluating probability distributions are Monte Carlo algorithms. Due to their tendency to sample low energy regions of conformational spaces, Monte Carlo-based algorithms are highly useful in finding important conformations of flexible biomolecules. With small adjustments, a Monte Carlo program can calculate a histogram of a distance distribution for a particle in harmonic potential. Such histograms illustrate that at any given temperature, the L-MTX and L-FMTX (with SWNTs \& BNNTs) distance adopts a range of values. It is also observed that the range of values gets broader with temperature, indicating increased amplitude of motion of atoms at higher temperatures. 
The effect of different solvents and temperatures on the L-MTX and L-FMTX (with SWNTs \& BNNTs) were studied through quantum mechanics calculations and molecular mechanic simulation. Differences in force fields were illustrated by comparing the energies calculated using AMBER, OPLS, CHARM (Bio+) and $\mathrm{MM}+$ force fields. Using this method, The Total (E tot), Potential (E pot) and Kinetic (E kin) energies $(\mathrm{kcal} / \mathrm{mol})$ were calculated for the Native structure through Monte Carlo simulation in different solvents and in AMBER, OPLS, CHARMM(Bio+) and MM+ force fields. The results have been listed in tables 2 to 9 .

Fig. 6-9 show the E Kin changes $(\mathrm{kcal} / \mathrm{mol})$ calculated versus temperature at different dielectric constants (water $(\varepsilon=78.39)$, DMSO $(\varepsilon=46.8)$, methanol ( $\varepsilon$ $=32.63)$, ethanol $(\varepsilon=24.55), \mathrm{CH} 2 \mathrm{Cl} 2(\varepsilon=8.93)$ and DMF $(\varepsilon=39.8))$ through Monte Carlo simulation in the four force fields (AMBER, OPLS, CHARMM (Bio+) and $\mathrm{MM}+$ ). The results of Monte Carlo calculations (tables 2-5 \& charts 6-11) indicate that in the gas phase and while in the Amber force field, L-MTX and L-FMTX connected to SWNTs and BNNTs are the most stable and have the lowest amount of energy. The methanol solvent displayed the lowest amount of energy and proved to be the most stable solvent for the simulation, when L-MTX connected to SWNTs was simulated in water, DMSO, methanol, ethanol, $\mathrm{CH} 2 \mathrm{Cl} 2$ and DMF solvents.

Similar results have been reported for OPLS and CHARMM force fields. The calculations related to the MM+ force field produced a notable result though. In this field, water was the most stable and the most suitable among the aforementioned solvents for simulation, since it had the lowest amount of energy. No doubt this was positively related to the dielectric constant of the solvents. Water had the highest dielectric constant; therefore, it is considered to be the most suitable solvent for L-MTX connected to SWNTs.

Substances with a high dielectric constant are easily polarized. Polarization allows countercharges to be placed around an ion, resulting in Coulombic interactions between the solvent and the ion, which in turn promote solubilization of the ion through competing with interionic interactions. In a similar vein, a polar solvent - one with a high dielectric constant - will form stabilizing interactions with the solute that compete with solute-solute interactions, thereby solubilizing polar molecules. The dielectric constant of the solvent also affects the interactions in the solution that involve ions and polar molecules; as the dielectric constant increases, the intermolecular energy is reduced.

It is noteworthy that tables 6-9 and charts 12-17, which display the results for L-FMTX connected to BNNTs, show that the results are highly consistent with those related to L-MTX connected to SWNTs; in the force fields Amber, OPLS and CHARMM, methanol is the most stable solvent and in the MM+ field, water is the most stable solvent.

On the other hand, water is a biological solvent and acts as the main foundation for chemical reactions. Results of chemical calculations can be influenced by solvation, which can push the simulation conditions toward the most stable form. However, the results for L-FMTX connected to BNNTs are very significant, since they are highly consistent with the behavior of SWNTs and point to methanol and water as being the most efficient solvents for this simulation. Given that performing calculations for Molecular Mechanics force fields requires selecting an appropriate force field in the beginning, the specifications of these 4 fields were closely investigated. Our choice was guided by force field equation for these fields.

Finally, we found that the MM+, which is an exclusive force field for calculations related to macromolecules, had the lowest amount of energy and featured the most stable form of connection for Methotrexate derivatives connected to SWNTs and BNNTs. Notably, in some solvents and at certain temperatures, the CHARMM force field demonstrates a similar behavior and puts our compound in a stable situation. However, since electrostatic reactions are calculated through bipolar junctions by using point charges in the MM+ field, the field managed to simulate our desired system in the most optimal way. Therefore, the MM+ was chosen as the most efficient force field. It should further be noted that the results of Quantum Mechanics calculations are also consistent with the current findings; SWNTs are more suitable carriers for L-MTX and BNNTs are more suitable carriers for L-FMTX. The results of Monte Carlo, Molecular Mechanics and Quantum Mechanics calculations have been justified.

Table 2. The Total (E tot), Potential (E pot) and Kinetic (E kin) energies (kcal/mol) calculated for the Native structure through Monte Carlo simulation in different solvents in the Amber force field (L-MTX)

\begin{tabular}{|c|c|c|c|c|c|c|c|c|c|c|c|}
\hline \multicolumn{12}{|c|}{ Monte Carlo.Amber } \\
\hline \multicolumn{2}{|c|}{ Temperature } & 298K & $300 \mathrm{~K}$ & 302K & $304 K$ & $306 \mathrm{~K}$ & 308K & $310 K$ & $312 K$ & $314 K$ & 316K \\
\hline \multirow{3}{*}{$\begin{array}{c}\text { Gas } \\
\left(\varepsilon_{\mathrm{r}}=1\right)\end{array}$} & E kin & 369.5223 & 372.0023 & 374.4823 & 376.9623 & 379.4423 & 381.9223 & 384.4024 & 386.8824 & 389.3624 & 391.8424 \\
\hline & E pot & 1215035 & 94875.69 & 18072.93 & 4770.186 & 1891.809 & 977.8239 & 596.4858 & 386.4485 & 288.5021 & 230.005 \\
\hline & E tot & 1215405 & 95247.69 & 18447.41 & 5147.148 & 2271.251 & 1359.746 & 980.8882 & 773.3309 & 677.8645 & 621.8474 \\
\hline \multirow{3}{*}{$\begin{array}{c}\text { Water } \\
\left(\varepsilon_{\mathrm{r}}=78.39\right)\end{array}$} & E kin & 1632.649 & 1643.606 & 1654.564 & 1665.521 & 1676.478 & 1687.436 & 1698.393 & 1709.35 & 1720.308 & 1731.265 \\
\hline & E pot & 1107903 & 100680.9 & 27634.59 & 14565.76 & 10818.86 & 9247.115 & 8228.382 & 7479.221 & 7042.025 & 6654.092 \\
\hline & E tot & 1109536 & 102324.5 & 29289.16 & 16231.28 & 12495.34 & 10934.55 & 9926.775 & 9188.571 & 8762.333 & 8385.357 \\
\hline \multirow{3}{*}{$\begin{array}{l}\text { Methanol } \\
\left(\varepsilon_{\mathrm{r}}=32.63\right)\end{array}$} & E kin & 636.0047 & 640.2732 & 644.5416 & 648.8101 & 653.0786 & 657.3471 & 661.6156 & 665.8841 & 670.1526 & 674.4211 \\
\hline & E pot & 1245003 & 114642.2 & 24691.49 & 7086.969 & 2992.702 & 1800.709 & 1247.194 & 913.8457 & 695.4784 & 575.4344 \\
\hline & E tot & 1245639 & 115282.4 & 25336.04 & 7735.779 & 3645.78 & 2458.056 & 1908.81 & 1579.73 & 1365.631 & 1249.855 \\
\hline \multirow{3}{*}{$\begin{array}{c}\text { Ethanol } \\
\left(\varepsilon_{\mathrm{r}}=\mathbf{2 4 . 5 5}\right)\end{array}$} & E kin & 769.2459 & 774.4086 & 779.5713 & 784.734 & 789.8968 & 795.0595 & 800.2222 & 805.3849 & 810.5477 & 815.7104 \\
\hline & E pot & 1203230 & 108701.5 & 22846.09 & 6582.576 & 2828.231 & 1709.052 & 1221.028 & 1001.106 & 880.3172 & 732.2387 \\
\hline & E tot & 1203999 & 109475.9 & 23625.66 & 7367.31 & 3618.128 & 2504.111 & 2021.251 & 1806.491 & 1690.865 & 1547.949 \\
\hline \multirow{3}{*}{$\begin{array}{c}\text { DMSO } \\
(\varepsilon r=46.8)\end{array}$} & E kin & 813.6596 & 819.1204 & 824.5812 & 830.042 & 835.5028 & 840.9636 & 846.4244 & 851.8852 & 857.346 & 862.8068 \\
\hline & E pot & 1342842 & 144844.7 & 35076.15 & 10938.13 & 4311.248 & 2199.194 & 1498.577 & 1100.033 & 949.6149 & 810.0334 \\
\hline & E tot & 1363655 & 145663.8 & 35900.73 & 11768.18 & 5146.751 & 3040.157 & 2345.001 & 1951.918 & 1806.961 & 1672.84 \\
\hline \multirow{3}{*}{$\begin{array}{c}\text { DMF } \\
(\varepsilon \mathrm{r}=38.3)\end{array}$} & E kin & 881.1685 & 887.0824 & 892.9962 & 898.9101 & 904.824 & 910.7379 & 916.6518 & 922.5657 & 928.4795 & 934.3934 \\
\hline & E pot & 1553272 & 168777.7 & 45394.16 & 16272.58 & 7950.383 & 4364.089 & 2774.878 & 2017.792 & 1619.377 & 1274.044 \\
\hline & E tot & 1554153 & 169664.8 & 46287.15 & 17171.49 & 8855.207 & 5274.826 & 3691.53 & 2940.358 & 2547.856 & 2208.438 \\
\hline
\end{tabular}


Table 3. The Total (E tot), Potential (E pot) and Kinetic (E kin) energies (kcal/mol) calculated for the Native structure through Monte Carlo simulation in different solvents in the Opls force field (L-MTX with SWNTs)

\begin{tabular}{|c|c|c|c|c|c|c|c|c|c|c|c|}
\hline \multicolumn{12}{|c|}{ Monte Carlo. Opls } \\
\hline \multicolumn{2}{|c|}{ Temperature } & $298 K$ & $300 \mathrm{~K}$ & $302 K$ & $304 K$ & $306 K$ & $308 K$ & $310 K$ & 312K & $314 K$ & $316 K$ \\
\hline \multirow{3}{*}{$\begin{array}{c}\text { Gas } \\
\left(\varepsilon_{\mathrm{r}}=1\right)\end{array}$} & E kin & 369.5223 & 372.0023 & 374.4823 & 376.9623 & 379.4423 & 381.9223 & 384.4024 & 386.8824 & 389.3624 & 391.8424 \\
\hline & E pot & 1772265 & 166731.1 & 33535.61 & 8798.063 & 3279.649 & 1805.931 & 1152.415 & 863.3952 & 674.8464 & 541.0597 \\
\hline & E tot & 1772634 & 167103.1 & 33910.09 & 9175.026 & 3659.091 & 2187.853 & 1536.817 & 1250.278 & 1064.209 & 932.9021 \\
\hline \multirow{3}{*}{$\begin{array}{c}\text { Water } \\
\left(\varepsilon_{\mathrm{r}}=78.39\right)\end{array}$} & E kin & 1632.649 & 1643.606 & 1654.564 & 1665.521 & 1676.478 & 1687.436 & 1698.393 & 1709.35 & 1720.308 & 1731.265 \\
\hline & E pot & 2083885 & 183886.4 & 63832.85 & 38714.33 & 30203.93 & 25987.91 & 23566.26 & 21998.69 & 20839.26 & 19754.51 \\
\hline & E tot & 2085518 & 185530 & 65487.41 & 40379.85 & 31880.41 & 27675.35 & 25264.65 & 23708.04 & 22559.56 & 21485.77 \\
\hline \multirow{3}{*}{$\begin{array}{c}\text { Methanol } \\
\left(\varepsilon_{\mathrm{r}}=32.63\right)\end{array}$} & E kin & 636.0047 & 640.2732 & 644.5416 & 648.8101 & 653.0786 & 657.3471 & 661.6156 & 665.8841 & 670.1526 & 674.4211 \\
\hline & E pot & 2110145 & 198664.3 & 43810.95 & 12732.32 & 5276.441 & 3001.545 & 2092.551 & 1575.327 & 1280.798 & 1130.354 \\
\hline & E tot & 2110781 & 199304.5 & 44455.49 & 13381.13 & 5929.52 & 3658.892 & 2754.166 & 2241.211 & 1950.951 & 1804.775 \\
\hline \multirow{3}{*}{$\begin{array}{c}\text { Ethanol } \\
\left(\varepsilon_{\mathrm{r}}=24.55\right)\end{array}$} & E kin & 76902459 & 774.4086 & 779.5713 & 784.734 & 789.8968 & 795.0595 & 800.2222 & 805.3849 & 810.5477 & 815.7104 \\
\hline & E pot & 2073174 & 158448.3 & 40652.37 & 11500.14 & 4368.956 & 2650.884 & 1725.141 & 1380.603 & 1140.458 & 958.1391 \\
\hline & E tot & 2073943 & 186222.7 & 41431.95 & 12284.88 & 5158.853 & 3355.943 & 2525.363 & 2185.985 & 1951.005 & 1773.849 \\
\hline \multirow{3}{*}{$\begin{array}{c}\text { DMSO } \\
(\varepsilon r=46.8)\end{array}$} & E kin & 813.6569 & 819.1204 & 824.5812 & 830.042 & 835.5028 & 840.9636 & 846.4244 & 851.8852 & 857.3460 & 862.8068 \\
\hline & E pot & 2309454 & 226424.08 & 55216.16 & 15722.95 & 5772.276 & 3009.73 & 2037.231 & 1583.689 & 1262.06 & 1093.549 \\
\hline & E tot & 2310268 & 227243.9 & 56040.75 & 16553 & 6607.258 & 3850.693 & 2883.626 & 2435.574 & 2119.406 & 1656.356 \\
\hline \multirow{3}{*}{$\begin{array}{c}\text { DMF } \\
(\varepsilon r=38.3)\end{array}$} & E kin & 881.1685 & 877.0824 & 892.9956 & 898.9102 & 904.824 & 910.7379 & 916.6518 & 992.5657 & 928.4795 & 934.3943 \\
\hline & E pot & 2373464 & 273300.9 & 76129.45 & 27600.37 & 12226.23 & 6033.796 & 3608.733 & 2427.096 & 1844.653 & 1498.755 \\
\hline & E tot & 2374346 & 274188 & 77022.45 & 28499.28 & 13130.84 & 6944.534 & 4525.385 & 3349.662 & 2773.132 & 2433.148 \\
\hline
\end{tabular}

Table 4. The Total (E tot), Potential (E pot) and Kinetic (E kin) energies (kcal/mol) calculated for the Native structure through Monte Carlo simulation in different solvents in the CHARMM force field (L-MTX with SWNTs)

\begin{tabular}{|c|c|c|c|c|c|c|c|c|c|c|c|}
\hline \multicolumn{12}{|c|}{ Monte Carlo.Charmm } \\
\hline \multicolumn{2}{|c|}{ Temperature } & \multirow{2}{*}{$\begin{array}{c}\mathbf{2 9 8 K} \\
369.5223\end{array}$} & \multirow{2}{*}{$\begin{array}{c}\text { 300K } \\
372.0023\end{array}$} & \multirow{2}{*}{$\begin{array}{c}\text { 302K } \\
374.4823\end{array}$} & \multirow{2}{*}{$\begin{array}{c}\text { 304K } \\
376.9623\end{array}$} & \multirow{2}{*}{$\begin{array}{c}\text { 306K } \\
379.4423\end{array}$} & \multirow{2}{*}{$\begin{array}{c}\mathbf{3 0 8 K} \\
381.9223\end{array}$} & \multirow{2}{*}{$\begin{array}{c}\text { 310K } \\
384.4024\end{array}$} & \multirow{2}{*}{$\begin{array}{c}\text { 312K } \\
386.8824\end{array}$} & \multirow{2}{*}{$\begin{array}{c}\text { 314K } \\
389.3624\end{array}$} & \multirow{2}{*}{$\begin{array}{c}\text { 316K } \\
391.8424\end{array}$} \\
\hline & E kin & & & & & & & & & & \\
\hline (c) & E pot & 571579.5 & 49052.96 & 9449.037 & 2750.36 & 1361.871 & 856.5552 & 599.3778 & 446.3805 & 343.2243 & 305.3477 \\
\hline & E tot & 571949 & 49424.96 & 9823.35 & 3127.323 & 1741.313 & 1238.478 & 983.7802 & 833.2629 & 732.5867 & 697.1901 \\
\hline \multirow{3}{*}{$\begin{array}{c}\text { Water } \\
\left(\varepsilon_{\mathrm{r}}=\mathbf{7 8 . 3 9}\right)\end{array}$} & E kin & 1632.649 & 1643.606 & 1654.564 & 1665.521 & 1676.478 & 1687.436 & 1698.393 & 1709.35 & 1720.308 & 1731.265 \\
\hline & E pot & 618115.4 & 51317.69 & 11306.14 & 4102.412 & 1577.696 & 374.0644 & -329.8485 & -867.5709 & -1247.758 & -1462.535 \\
\hline & E tot & 619748 & 52961.3 & 12960.71 & 5767.933 & 3254.175 & 2061.5 & 1368.545 & 841.7796 & 472.5496 & 268.7304 \\
\hline \multirow{3}{*}{$\begin{array}{l}\text { Methanol } \\
\left(\varepsilon_{\mathrm{r}}=32.63\right)\end{array}$} & E kin & 636.0047 & 640.2732 & 644.5416 & 648.8101 & 653.0786 & 657.3471 & 661.6156 & 665.8841 & 670.1526 & 674.4211 \\
\hline & E pot & 662941.2 & 61217.51 & 13531.14 & 4606.004 & 2491.551 & 1617.565 & 1223.629 & 981.8296 & 825.4841 & 703.4496 \\
\hline & E tot & 663577.2 & 61857.79 & 14175.68 & 5254.814 & 3144.63 & 2274.912 & 1885.244 & 1647.714 & 1495.637 & 1377.871 \\
\hline \multirow{3}{*}{$\begin{array}{c}\text { Ethanol } \\
\left(\varepsilon_{\mathrm{r}}=\mathbf{2 4 . 5 5}\right)\end{array}$} & E kin & 769.2459 & 774.4086 & 779.5713 & 784.734 & 789.8968 & 795.0595 & 800.2222 & 805.3849 & 810.5477 & 815.7104 \\
\hline & E pot & 662354.4 & 62431.12 & 13415.3 & 4372.998 & 2223.693 & 1486.936 & 1229.257 & 946.9232 & 800.0959 & 731.11 \\
\hline & E tot & 663123.6 & 63205.52 & 14194.87 & 5157.732 & 3013.59 & 2281.996 & 1929.479 & 1752.308 & 1610.644 & 1546.82 \\
\hline \multirow{3}{*}{$\begin{array}{c}\text { DMSO } \\
(\varepsilon \mathrm{r}=46.8)\end{array}$} & E kin & 813.6596 & 819.1204 & 824.5812 & 830.042 & 835.5028 & 840.9636 & 846.4244 & 851.8852 & 857.346 & 862.8068 \\
\hline & E pot & 766294.3 & 87140.02 & 21484.24 & 6906.317 & 2941.12 & 1886.499 & 1399.431 & 1104.159 & 995.512 & 872.8668 \\
\hline & E tot & 767108 & 87959.25 & 22309.25 & 7736.25 & 3776.2356 & 2727.325 & 2445.885 & 1956.044 & 1853.858 & 1735.675 \\
\hline \multirow{3}{*}{$\begin{array}{c}\text { DMF } \\
(\varepsilon \mathrm{r}=38.3)\end{array}$} & E kin & 881.1685 & 877.0824 & 892.9625 & 898.2581 & 904.4578 & 910.7379 & 916.2587 & 922.5686 & 928.2587 & 934.2101 \\
\hline & E pot & 905576 & 123315.01 & 33588.02 & 12112.03 & 5937.56 & 3511.025 & 2533.64 & 1958.25 & 1578.25 & 1374.045 \\
\hline & E tot & 906457.2 & 124202.01 & 34481.02 & 13011.25 & 6862.02 & 4421.0258 & 3450.025 & 2881.258 & 2507.25 & 2308.258 \\
\hline
\end{tabular}


J. Chil. Chem. Soc., 66, N $4(2021)$

Table 5. The Total (E tot), Potential (E pot) and Kinetic (E kin) energies (kcal/mol) calculated for the Native structure through Monte Carlo simulation in different solvents in the MM+ force field (L-MTX with SWNTs)

\begin{tabular}{|c|c|c|c|c|c|c|c|c|c|c|c|}
\hline \multicolumn{12}{|c|}{ Monte Carlo.MM+ } \\
\hline \multicolumn{2}{|c|}{ Temperature } & 298K & $300 \mathrm{~K}$ & $302 K$ & $304 K$ & $306 K$ & $308 K$ & $310 \mathrm{~K}$ & $312 K$ & $314 K$ & $316 K$ \\
\hline \multirow{3}{*}{$\begin{array}{c}\text { Gas } \\
\left(\varepsilon_{\mathrm{r}}=1\right)\end{array}$} & E kin & 369.5223 & 372.0023 & 374.4823 & 376.9623 & 379.4423 & 381.9223 & 384.4024 & 386.8824 & 389.3624 & 391.8424 \\
\hline & E pot & 54285.77 & 15756.75 & 6110.059 & 3043.559 & 1810.011 & 1215.896 & 898.1151 & 688.2434 & 553.4915 & 427.8488 \\
\hline & E tot & 54655.3 & 16128.75 & 6484.541 & 3420.521 & 2189.453 & 1597.818 & 1282.517 & 1075.126 & 942.8539 & 819.6912 \\
\hline \multirow{3}{*}{$\begin{array}{c}\text { Water } \\
\left(\varepsilon_{\mathrm{r}}=\mathbf{7 8 . 3 9}\right)\end{array}$} & E kin & 1632.258 & 1643.025 & 1654.890 & 1665.028 & 1676.981 & 1687.987 & 1698.214 & 1709.351 & 1720.921 & 1731.324 \\
\hline & E pot & 246270.3 & 19302.25 & 154461.1 & 128223.04 & 111018.25 & 97520.36 & 84963.25 & 73770.63 & 63986.02 & 55082.36 \\
\hline & E tot & 247903.12 & 194664.02 & 156116.31 & 129889.65 & 112695.23 & 99207.23 & 86661.23 & 75480.23 & 65706.21 & 56813.25 \\
\hline \multirow{3}{*}{$\begin{array}{l}\text { Methanol } \\
\left(\varepsilon_{\mathrm{r}}=32.63\right)\end{array}$} & E kin & 636.0047 & 640.2732 & 644.5416 & 648.8101 & 653.0786 & 657.3471 & 661.6156 & 665.8841 & 670.1526 & 674.4211 \\
\hline & E pot & 264320.2 & 209363.2 & 168875.6 & 142200.6 & 123731.7 & 109095.1 & 96174.95 & 84833.81 & 74129.24 & 64710.34 \\
\hline & E tot & 264956.2 & 210003.5 & 169520.2 & 142849.5 & 124384.8 & 109752.4 & 96836.56 & 85499.69 & 74799.4 & 65384.76 \\
\hline \multirow{3}{*}{$\begin{array}{c}\text { Ethanol } \\
\left(\varepsilon_{\mathrm{r}}=\mathbf{2 4 . 5 5}\right)\end{array}$} & E kin & 769.2546 & 774.4086 & 779.5713 & 784.734 & 789.2587 & 795.0595 & 800.2222 & 805.2347 & 810.7123 & 815.2174 \\
\hline & E pot & 251251.1 & 200183.9 & 161017.9 & 134599.7 & 117273.3 & 103198.2 & 89837.1 & 78091.2 & 67984.2 & 59233.2 \\
\hline & E tot & 252020.4 & 200958.3 & 161797.4 & 135384.4 & 118063.2 & 103993.6 & 90637.21 & 78896.21 & 68795.20 & 60049.25 \\
\hline \multirow{3}{*}{$\begin{array}{c}\text { DMSO } \\
(\varepsilon \mathrm{r}=\mathbf{4 6 . 8})\end{array}$} & E kin & 813.6596 & 819.1204 & 824.5812 & 830.042 & 835.5028 & 840.9636 & 846.4244 & 851.8852 & 875.346 & 862.8068 \\
\hline & E pot & 273355.4 & 218738.4 & 177119 & 148530.1 & 129651.9 & 113657.7 & 100138.7 & 87823.95 & 77045.2 & 67538.76 \\
\hline & E tot & 274169.1 & 219557.5 & 177943.6 & 149360.1 & 130487.4 & 114498.6 & 100928.1 & 88675.84 & 77902.55 & 68401.57 \\
\hline \multirow{3}{*}{$\begin{array}{c}\text { DMF } \\
(\varepsilon r=38.3)\end{array}$} & E kin & 881.1685 & 877.0824 & 892.9962 & 898.9101 & 904.824 & 910.7379 & 916.6518 & 922.5657 & 928.4795 & 934.3934 \\
\hline & E pot & 334050.6 & 226510.2 & 217634.9 & 183354.2 & 160342.7 & 142128.2 & 125601.4 & 110880.8 & 97296.04 & 85741.55 \\
\hline & E tot & 334931.8 & 267397.2 & 218527.9 & 184253.1 & 161247.5 & 143038.9 & 126518.1 & 111803.3 & 98224.52 & 86675.94 \\
\hline
\end{tabular}

\section{MONTE CARLO(AMBER)}

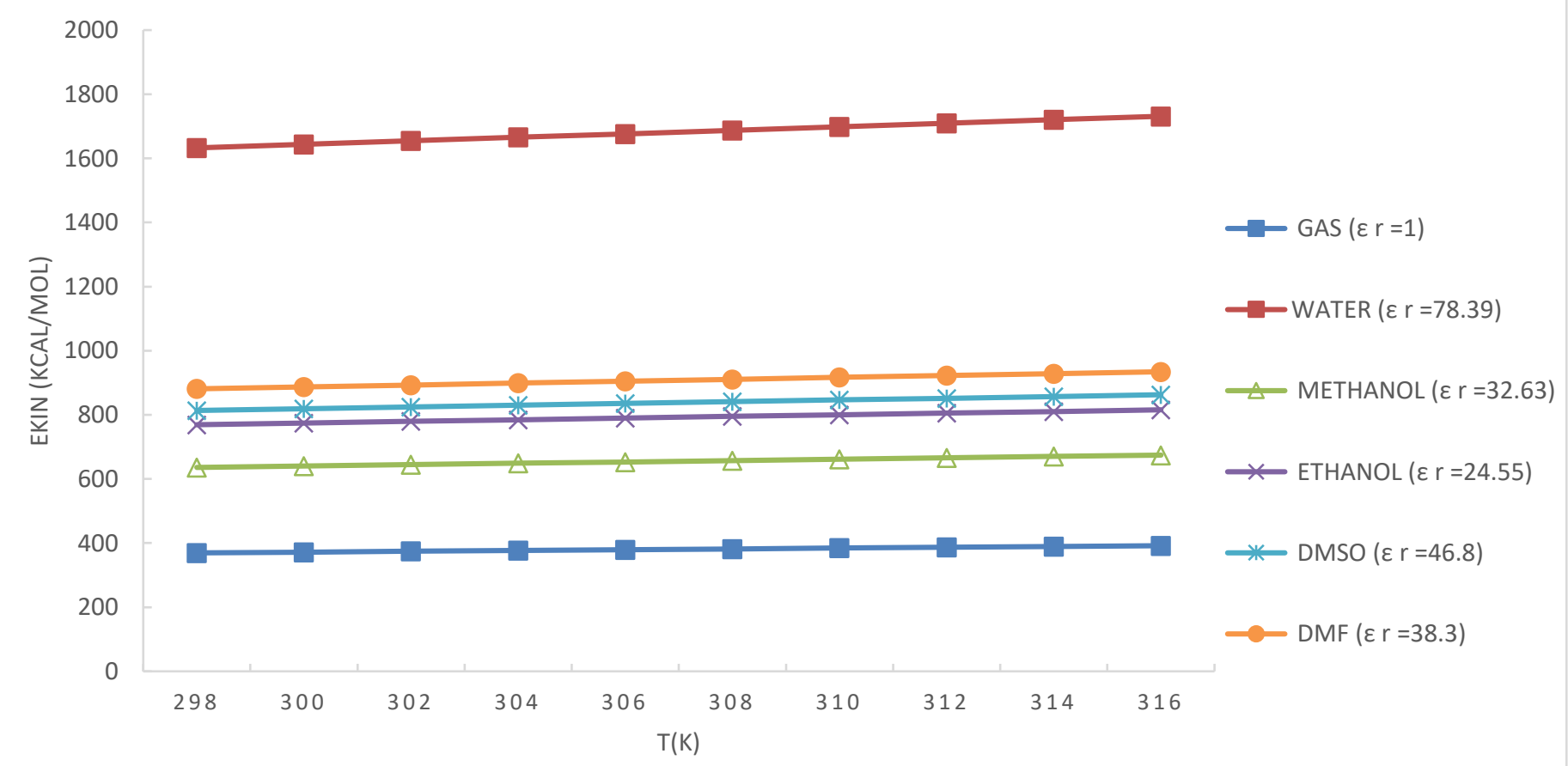

Figure 6. EKin changes (kcal.mol) calculated versus temperature at different dielectric constants through Monte Carlo simulation in the Amber force field for L-MTX with SWNTs. 


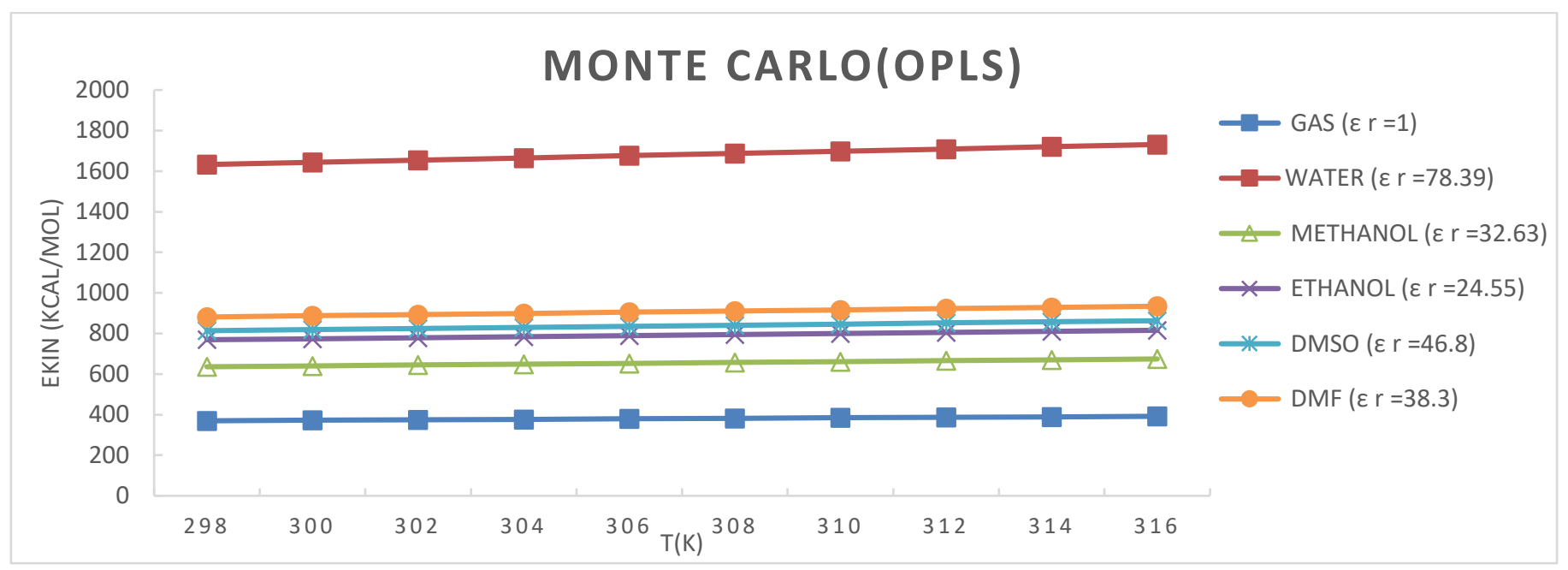

Figure 7. EKin changes (kcal.mol) calculated versus temperature at different dielectric constants through Monte Carlo simulation in the OPLS force field for L-MTX with SWNTs.

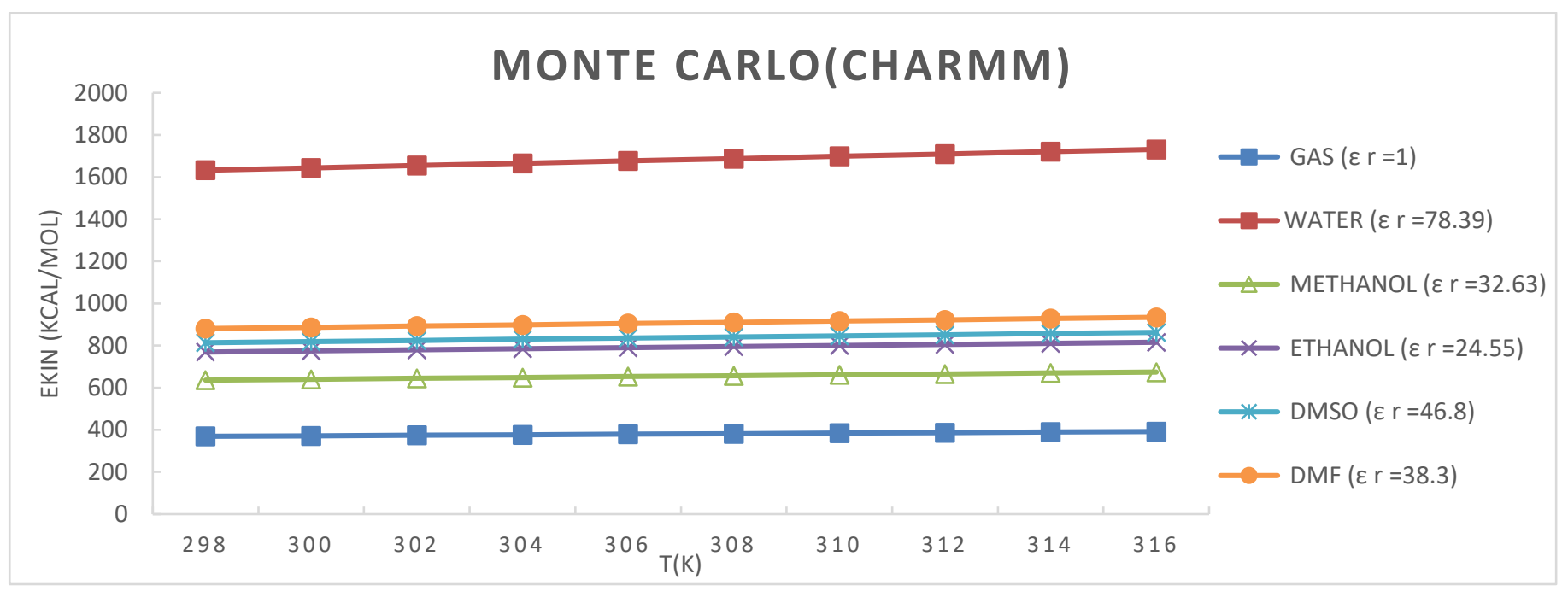

Figure 8. EKin changes (kcal.mol) calculated versus temperature at different dielectric constants through Monte Carlo simulation in the CHARMM force field for L-MTX with SWNTs.

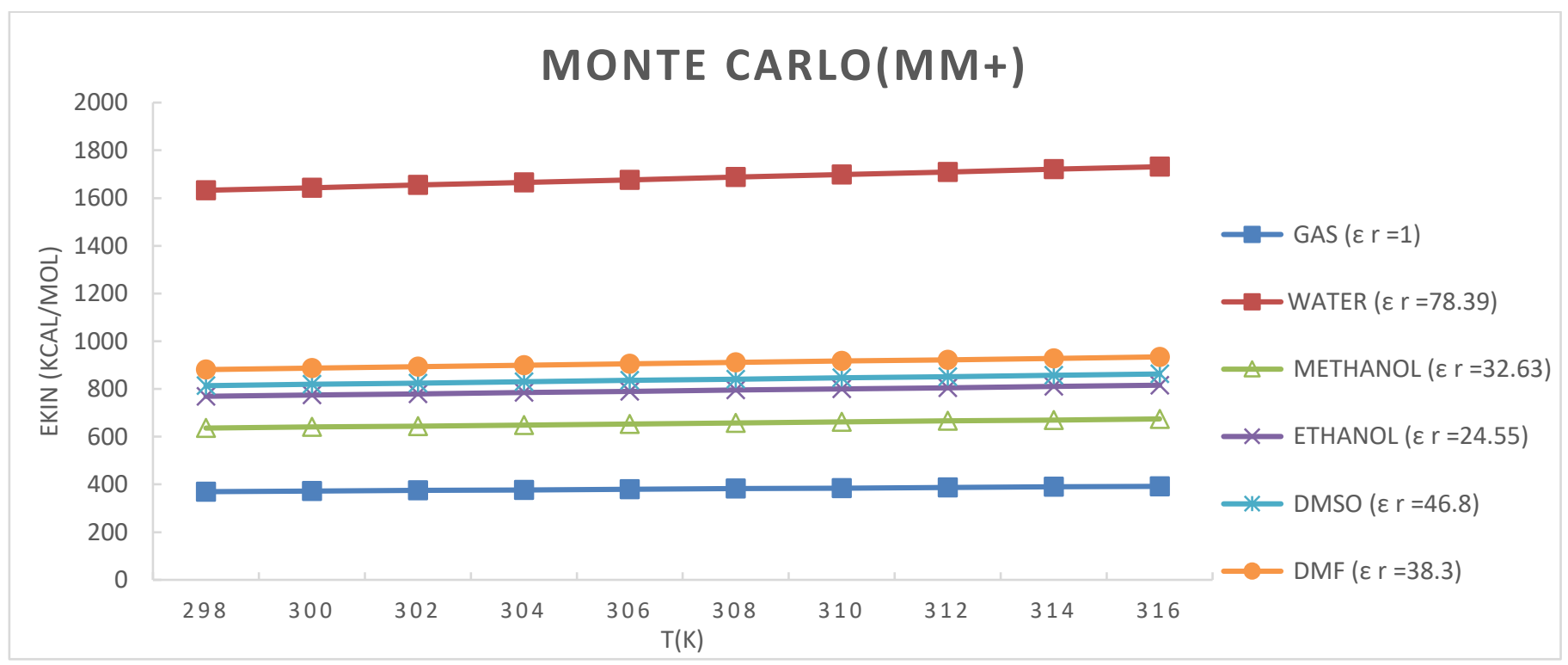

Figure 9. EKin changes (kcal.mol) calculated versus temperature at different dielectric constants through Monte Carlo simulation in the MM+ force field for L-MTX with SWNTs. 


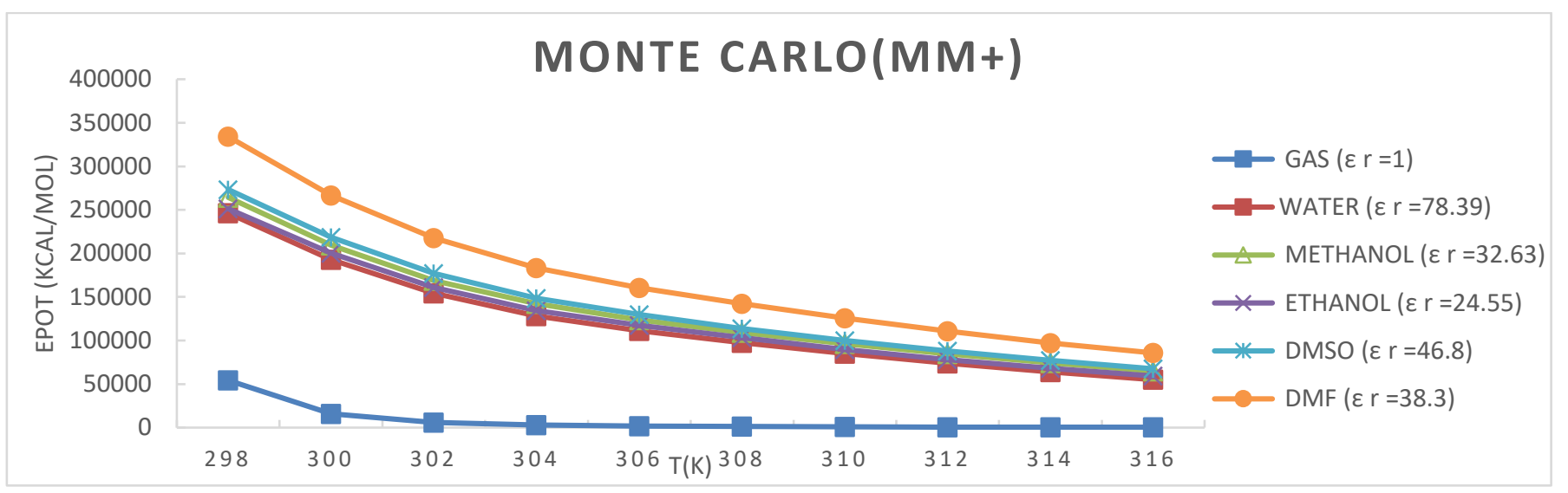

Figure 10. EPot changes (kcal.mol) calculated versus temperature at different dielectric constants through Monte Carlo simulation in the MM+ force field for L-MTX with SWNTs.

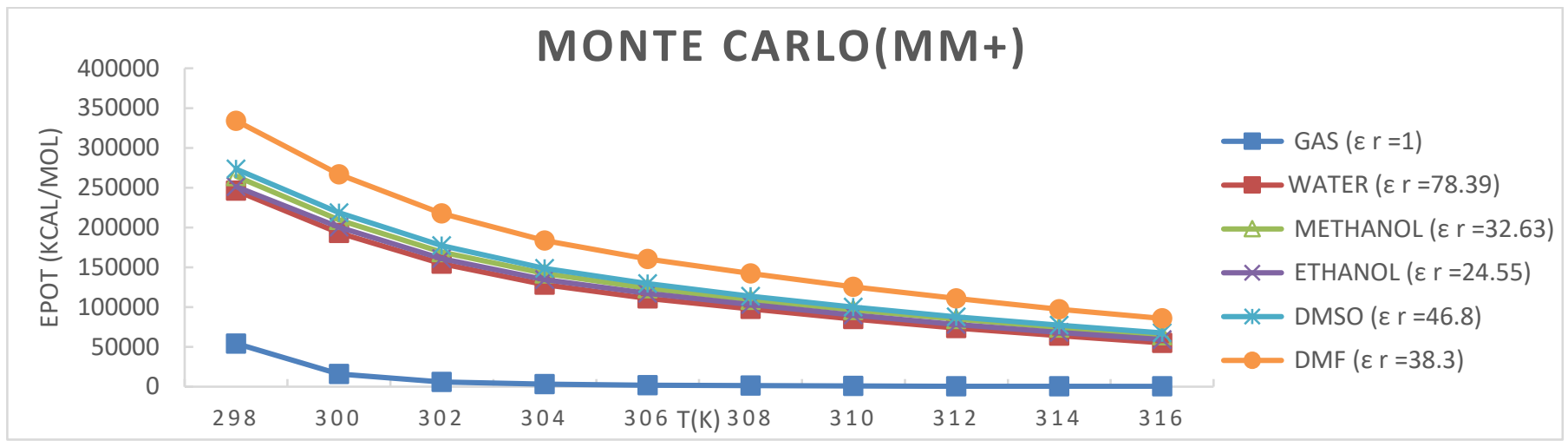

Figure 11. ETot changes (kcal.mol) calculated versus temperature at different dielectric constants through Monte Carlo simulation in the MM+ force field for L-MTX with SWNTs.

Table 6. The Total (E tot), Potential (E pot) and Kinetic (E kin) energies (kcal/mol) calculated for the Native structure through Monte Carlo simulation in different solvents in the Amber force field (L-FMTX with BNNTs)

\begin{tabular}{|c|c|c|c|c|c|c|c|c|c|c|c|}
\hline \multicolumn{12}{|c|}{ Monte Carlo.Amber } \\
\hline \multicolumn{2}{|c|}{ Temperature } & $298 K$ & $300 K$ & $302 K$ & $304 K$ & $306 K$ & 308K & $310 K$ & 312K & 314K & $316 K$ \\
\hline \multirow{3}{*}{$\begin{array}{c}\text { Gas } \\
\left(\varepsilon_{\mathrm{r}}=1\right)\end{array}$} & E kin & 369.5223 & 372.0023 & 374.4823 & 376.9623 & 379.4423 & 381.9223 & 384.4024 & 386.8824 & 389.3624 & 391.8424 \\
\hline & E pot & 1142735 & 94865.64 & 18303.79 & 4647.02 & 1834.974 & 972.0237 & 587.2981 & 394.5939 & 304.2547 & 197.403 \\
\hline & E tot & 1143104 & 95237.64 & 18678.27 & 5023.982 & 2214.416 & 1353.946 & 971.7005 & 781.4763 & 693.6171 & 589.2454 \\
\hline \multirow{3}{*}{$\begin{array}{c}\text { Water } \\
\left(\varepsilon_{\mathrm{r}}=78.39\right)\end{array}$} & E kin & 1629.984 & 1640.924 & 1651.863 & 1662.803 & 1673.742 & 1684.681 & 1695.621 & 1706.56 & 1717.5 & 1728.439 \\
\hline & E pot & 1096642 & 98711.55 & 26880.44 & 14338.82 & 10659.22 & 8864.739 & 7781.433 & 7141.914 & 6658.069 & 6314.386 \\
\hline & E tot & 1098272 & 100352.5 & 28532.3 & 16001.62 & 12332.96 & 10549.42 & 9477.054 & 8848.474 & 8375.569 & 8042.826 \\
\hline \multirow{3}{*}{$\begin{array}{c}\text { Metanol } \\
\left(\varepsilon_{\mathrm{r}}=32.63\right)\end{array}$} & E kin & 636.0047 & 640.2732 & 644.5416 & 648.8101 & 653.0786 & 657.3471 & 661.6156 & 665.8841 & 670.1526 & 674.4211 \\
\hline & E pot & 1279321 & 130933 & 30253.88 & 9665.178 & 4374.126 & 2548.176 & 1680.624 & 1166.651 & 980.7566 & 715.7875 \\
\hline & E tot & 1279957 & 131573.2 & 30898.42 & 10313.99 & 5027.205 & 3205.523 & 2342.239 & 1832.535 & 1650.909 & 1390.209 \\
\hline \multirow{3}{*}{$\begin{array}{c}\text { Ethanol } \\
\left(\varepsilon_{\mathrm{r}}=\mathbf{2 4 . 5 5}\right)\end{array}$} & E kin & 777.2403 & 782.4567 & 787.6731 & 792.8895 & 798.1059 & 803.3222 & 808.5386 & 813.755 & 818.9714 & 824.1878 \\
\hline & E pot & 1270246 & 107898 & 21995.52 & 6122.308 & 2672.688 & 1585.394 & 1153.461 & 858.065 & 773.4414 & 659.9271 \\
\hline & E tot & 1271023 & 108680.4 & 22783.19 & 6915.198 & 3470.794 & 2388.716 & 1961.999 & 1671.82 & 1592.413 & 1484.115 \\
\hline \multirow{3}{*}{$\begin{array}{c}\text { DMSO } \\
(\varepsilon r=46.8)\end{array}$} & E kin & 813.6596 & 819.1204 & 824.5812 & 830.042 & 835.5028 & 840.9636 & 846.4244 & 851.8852 & 857.346 & 862.8068 \\
\hline & E pot & 1359596 & 135193.8 & 30189.1 & 8644.713 & 3741.646 & 2148.814 & 1426.887 & 1093.304 & 870.8363 & 763.3856 \\
\hline & E tot & 1360409 & 136012.9 & 31013.68 & 9474.755 & 4577.149 & 2989.778 & 2273.311 & 1945.189 & 1728.182 & 1626.192 \\
\hline \multirow{3}{*}{$\begin{array}{c}\text { DMF } \\
(\varepsilon \mathrm{r}=\mathbf{3 8 . 3})\end{array}$} & E kin & 881.1685 & 887.0824 & 892.9962 & 898.9101 & 904.824 & 910.7379 & 916.6518 & 922.5657 & 928.4795 & 934.3934 \\
\hline & E pot & 1275730 & 114925.9 & 24774.87 & 6946.043 & 2883.357 & 1708.11 & 1200.27 & 1013.348 & 833.981 & 721.7818 \\
\hline & E tot & 1276611 & 115812.9 & 25667.87 & 7844.953 & 3789.182 & 2618.848 & 2116.922 & 1935.913 & 1762.461 & 1656.265 \\
\hline
\end{tabular}


Table 7. The Total (E tot), Potential (E pot) and Kinetic (E kin) energies (kcal/mol) calculated for the Native structure through Monte Carlo simulation in different solvents in the OPLS force field (L-FMTX with BNNTs)

\begin{tabular}{|c|c|c|c|c|c|c|c|c|c|c|c|}
\hline \multicolumn{12}{|c|}{ Monte Carlo.OPLS } \\
\hline \multicolumn{2}{|c|}{ Temperature } & 298K & $300 \mathrm{~K}$ & 302K & $304 K$ & $306 K$ & $308 K$ & $310 K$ & $312 K$ & $314 K$ & $316 K$ \\
\hline \multirow{3}{*}{$\begin{array}{c}\text { Gas } \\
\left(\varepsilon_{\mathrm{r}}=1\right)\end{array}$} & E kin & 369.5223 & 372.0023 & 374.4823 & 376.9623 & 379.4423 & 381.9223 & 384.4024 & 386.8824 & 389.3624 & 391.8424 \\
\hline & E pot & 1965648 & 164206.9 & 33649.14 & 9358.676 & 3457.705 & 1769.1 & 1158.373 & 830.4087 & 633.6891 & 496.2554 \\
\hline & E tot & 1966017 & 164578.9 & 34023.62 & 9735.639 & 3837.147 & 2151.023 & 1542.775 & 1217.291 & 1023.051 & 888.0978 \\
\hline \multirow{3}{*}{$\begin{array}{c}\text { Water } \\
\left(\varepsilon_{\mathrm{r}}=\mathbf{7 8 . 3 9}\right)\end{array}$} & E kin & 1629.984 & 1640.924 & 1651.863 & 1662.803 & 1673.742 & 1684.681 & 1695.621 & 1706.56 & 1717.5 & 1728.439 \\
\hline & E pot & 2094370 & 198506 & 64325.85 & 38926.22 & 30434.71 & 25968.6 & 23209.8 & 21298.77 & 19912.09 & 18889.24 \\
\hline & E tot & 2096000 & 200146.9 & 65977.72 & 40589.02 & 32108.45 & 27653.28 & 24905.42 & 23005.33 & 21629.59 & 20617.68 \\
\hline \multirow{3}{*}{$\begin{array}{l}\text { Methanol } \\
\left(\varepsilon_{\mathrm{r}}=32.63\right)\end{array}$} & E kin & 636.0047 & 640.2732 & 644.5416 & 648.8101 & 653.0786 & 657.3471 & 661.6156 & 665.8841 & 670.1526 & 674.4211 \\
\hline & E pot & 2343471 & 230921.9 & 55124.66 & 16652.99 & 7053.134 & 3920.395 & 2570.881 & 1883.181 & 1517.791 & 1280.233 \\
\hline & E tot & 2344107 & 231562.2 & 55769.2 & 17301.81 & 7706.213 & 4577.742 & 3232.497 & 2549.065 & 2187.944 & 1954.654 \\
\hline \multirow{3}{*}{$\begin{array}{c}\text { Ethanol } \\
\left(\varepsilon_{\mathrm{r}}=\mathbf{2 4 . 5 5}\right)\end{array}$} & E kin & 777.2403 & 782.4567 & 787.6731 & 792.8895 & 798.1059 & 803.3222 & 808.5386 & 813.755 & 818.9714 & 824.1878 \\
\hline & E pot & 2889709 & 269417.9 & 68568.48 & 19707.84 & 7369.325 & 3870.296 & 2523.986 & 1833.309 & 1468.068 & 1183.452 \\
\hline & E tot & 2890486 & 270200.4 & 69536.15 & 20500.73 & 8167.431 & 4673.618 & 3332.525 & 2647.064 & 2287.039 & 2007.639 \\
\hline \multirow{3}{*}{$\begin{array}{c}\text { DMSO } \\
(\varepsilon r=46.8)\end{array}$} & E kin & 813.6596 & 819.1204 & 824.5812 & 830.042 & 835.5028 & 840.9636 & 846.4244 & 851.8852 & 857.346 & 862.8068 \\
\hline & E pot & 2373698 & 216770.4 & 51601.83 & 15087.13 & 5974.574 & 3397.155 & 2180.909 & 1619.642 & 1383.075 & 1182.843 \\
\hline & E tot & 2374511 & 217589.5 & 52624.41 & 15917.17 & 6810.076 & 4238.119 & 3027.333 & 2543.527 & 2240.421 & 2045.65 \\
\hline \multirow{3}{*}{$\begin{array}{c}\text { DMF } \\
(\varepsilon \mathrm{r}=\mathbf{3 8 . 3})\end{array}$} & E kin & 881.1685 & 887.0824 & 892.9962 & 898.9101 & 904.824 & 910.7379 & 916.6518 & 922.5657 & 928.4795 & 934.3934 \\
\hline & E pot & 2203015 & 197073 & 42786.12 & 12371.11 & 4913.181 & 2722.508 & 1873.174 & 1428.213 & 1210.222 & 1059.561 \\
\hline & E tot & 2203896 & 197960 & 43679.11 & 13270.02 & 5818.005 & 3633.264 & 2789.826 & 2350.779 & 2138.702 & 1993.995 \\
\hline
\end{tabular}

Table 8. The Total (E tot), Potential (E pot) and Kinetic (E kin) energies (kcal/mol) calculated for the Native structure through Monte Carlo Simulation in different solvents in the CHARMM force field (L-FMTX with BNNTs)

\begin{tabular}{|c|c|c|c|c|c|c|c|c|c|c|c|}
\hline \multicolumn{12}{|c|}{ Monte Carlo.Charmm } \\
\hline \multicolumn{2}{|l|}{ Temperature } & 298K & $300 K$ & 302K & 304K & $306 K$ & $308 K$ & $310 K$ & $312 K$ & $314 K$ & $316 K$ \\
\hline \multirow{3}{*}{$\begin{array}{c}\text { Gas } \\
\left(\varepsilon_{\mathrm{r}}=1\right)\end{array}$} & E kin & 369.5223 & 372.0023 & 374.4823 & 376.9623 & 379.4423 & 381.9223 & 384.4024 & 386.8824 & 389.3624 & 391.8424 \\
\hline & E pot & 586516.9 & 49778.8 & 9861.559 & 2957.149 & 1391.578 & 797.6654 & 524.1795 & 419.3601 & 349.0755 & 263.6077 \\
\hline & E tot & 586886.4 & 50150.8 & 10236.04 & 3334.111 & 1771.02 & 1179.588 & 908.5819 & 806.2425 & 738.4379 & 655.4501 \\
\hline \multirow{3}{*}{$\begin{array}{c}\text { Water } \\
\left(\varepsilon_{\mathrm{r}}=\mathbf{7 8 . 3 9}\right)\end{array}$} & E kin & 1629.984 & 1640.924 & 1651.863 & 1662.803 & 1673.742 & 1684.681 & 1695.621 & 1706.56 & 1717.5 & 1728.439 \\
\hline & E pot & 580286 & 50015.74 & 10822.28 & 4045.975 & 1632.549 & 376.2494 & -408.6071 & -919.0168 & -1278.327 & -1530.636 \\
\hline & E tot & 581915.9 & 51656.67 & 12474.14 & 5708.778 & 3306.291 & 2060.931 & 1287.014 & 787.5437 & 439.1726 & 197.8038 \\
\hline \multirow{3}{*}{$\begin{array}{c}\text { Methanol } \\
\left(\varepsilon_{\mathrm{r}}=32.63\right)\end{array}$} & E kin & 636.0047 & 640.2732 & 644.5416 & 648.8101 & 653.0786 & 657.3471 & 661.6156 & 665.8841 & 670.1526 & 674.4211 \\
\hline & E pot & 738310.8 & 81171.33 & 20200.17 & 6862.643 & 3291.717 & 1956.663 & 1432.668 & 1085.132 & 907.6039 & 743.0048 \\
\hline & E tot & 738946.8 & 81811.6 & 20844.71 & 7511.453 & 3944.796 & 2614.01 & 2094.284 & 1751.016 & 1577.756 & 1417.426 \\
\hline \multirow{3}{*}{$\begin{array}{c}\text { Ethanol } \\
\left(\varepsilon_{\mathrm{r}}=\mathbf{2 4 . 5 5}\right)\end{array}$} & E kin & 777.2403 & 782.4567 & 787.6731 & 792.8895 & 798.1059 & 803.3222 & 808.5368 & 813.755 & 818.9714 & 824.1878 \\
\hline & E pot & 986643.3 & 126666.6 & 32740.86 & 10776.3 & 5001.039 & 3054.866 & 2249.027 & 1675.546 & 1369.627 & 1228.446 \\
\hline & E tot & 987420.5 & 127449.1 & 33528.53 & 11569.19 & 5799.144 & 3858.189 & 3057.566 & 2489.301 & 2188.559 & 2052.633 \\
\hline \multirow{3}{*}{$\begin{array}{c}\text { DMSO } \\
(\varepsilon \mathrm{r}=46.8)\end{array}$} & E kin & 813.6596 & 819.1204 & 824.5812 & 830.42 & 835.5028 & 840.9636 & 864.4244 & 851.8552 & 857.346 & 862.8068 \\
\hline & E pot & 716422.8 & 79379.78 & 17109.43 & 5419.058 & 2856.074 & 1866.675 & 1355.04 & 1162.605 & 1011.852 & 880.7916 \\
\hline & E tot & 717236.4 & 80196.9 & 17934.01 & 6249.1 & 3691.577 & 2707.639 & 2201.465 & 2014.49 & 1869.198 & 1743.598 \\
\hline \multirow{3}{*}{$\begin{array}{c}\text { DMF } \\
(\varepsilon \mathrm{r}=38.3)\end{array}$} & E kin & 881.1685 & 887.0824 & 892.9262 & 898.9101 & 904.824 & 910.7379 & 916.6518 & 922.5657 & 928.4795 & 934.3934 \\
\hline & E pot & 660813.8 & 64232.28 & 13891.77 & 4512.75 & 2467.091 & 1727.232 & 1401.041 & 1251.961 & 1184.178 & 1069.467 \\
\hline & E tot & 661694.9 & 65119.36 & 14784.77 & 5411.66 & 3371.915 & 2637.97 & 2317.693 & 2174.527 & 2112.657 & 2003.86 \\
\hline
\end{tabular}


Table 9. The Total (E tot), Potential (E pot) and Kinetic (E kin) energies (kcal/mol) calculated for the Native structure through Monte Carlo simulation in different solvents in the MM+ force field (L-FMTX with BNNTs)

\begin{tabular}{|c|c|c|c|c|c|c|c|c|c|c|c|}
\hline \multicolumn{12}{|c|}{ Monte Carlo.MM+ } \\
\hline \multicolumn{2}{|c|}{ Temperature } & $298 K$ & $300 \mathrm{~K}$ & 302K & $304 K$ & $306 \mathrm{~K}$ & $308 K$ & $310 K$ & 312K & $314 K$ & $316 K$ \\
\hline \multirow{3}{*}{$\begin{array}{c}\text { Gas } \\
\left(\varepsilon_{\mathrm{r}}=1\right)\end{array}$} & E kin & 191.8673 & 193.155 & 194.4427 & 195.7304 & 197.0181 & 198.3058 & 199.5935 & 200.8812 & 202.1689 & 203.4566 \\
\hline & E pot & 1479.404 & 655.6518 & 492.8714 & 489.8405 & 470.9847 & 460.0471 & 461.0492 & 424.7249 & 432.992 & 441.904 \\
\hline & E tot & 1671.271 & 848.8069 & 687.3142 & 685.5709 & 668.0028 & 658.3529 & 660.6427 & 625.6061 & 635.161 & 645.3607 \\
\hline \multirow{3}{*}{$\begin{array}{c}\text { Water } \\
\left(\varepsilon_{\mathrm{r}}=78.39\right)\end{array}$} & E kin & 1561.587 & 1572.067 & 1582.548 & 1593.028 & 197.0181 & 1613.989 & 1624.47 & 1634.95 & 1645.43 & 1655.911 \\
\hline & E pot & 18688.64 & 11972.32 & 7590.633 & 5262.345 & 4107.78 & 3259.293 & 2599.388 & 2179.092 & 1811.395 & 1571.224 \\
\hline & E tot & 2025.33 & 13544.38 & 9173.18 & 7055.374 & 5711.289 & 4873.282 & 4223.585 & 3814.043 & 3456.828 & 3227.134 \\
\hline \multirow{3}{*}{$\begin{array}{l}\text { Methanol } \\
\left(\varepsilon_{\mathrm{r}}=32.63\right)\end{array}$} & E kin & 458.3497 & 461.4259 & 464.5021 & 467.5783 & 470.6544 & 473.7306 & 476.8068 & 479.8829 & 482.9591 & 486.0353 \\
\hline & E pot & 40507.78 & 29290.68 & 22471.8 & 17951.32 & 14028.16 & 11554.74 & 9531.721 & 8179.061 & 7063.156 & 6388.415 \\
\hline & E tot & 40966.13 & 29752.11 & 22936.3 & 18418.89 & 14498.81 & 12028.47 & 10008.53 & 8658.944 & 7546.115 & 6874.451 \\
\hline \multirow{3}{*}{$\begin{array}{c}\text { Ethanol } \\
\left(\varepsilon_{\mathrm{r}}=\mathbf{2 4 . 5 5}\right)\end{array}$} & E kin & 591.5909 & 595.5613 & 599.5318 & 603.5022 & 607.4726 & 611.443 & 615.4134 & 619.3838 & 623.3542 & 627.3246 \\
\hline & E pot & 32385.32 & 24101.97 & 18567.27 & 14918.58 & 12081.58 & 10105.09 & 8474.525 & 7332.273 & 6389.992 & 5672.525 \\
\hline & E tot & 32976.92 & 24697.53 & 19166.81 & 15522.08 & 12689.06 & 10716.53 & 9089.938 & 7951.657 & 7013.349 & 6292.859 \\
\hline \multirow{3}{*}{$\begin{array}{c}\text { DMSO } \\
(\varepsilon \mathrm{r}=46.8)\end{array}$} & E kin & 636.0047 & 640.2732 & 644.5416 & 648.8101 & 653.0776 & 657.3471 & 661.6156 & 665.8841 & 670.1526 & 674.4211 \\
\hline & E pot & 278379.1 & 221019.9 & 185756.2 & 158494.7 & 137388.8 & 121422 & 108744.2 & 98028.74 & 88391.26 & 80790.44 \\
\hline & E tot & 279015.1 & 221660.1 & 186400.7 & 159143.5 & 138041.9 & 122079.4 & 109405.8 & 98694.23 & 89061.41 & 81464.86 \\
\hline \multirow{3}{*}{$\begin{array}{c}\text { DMF } \\
(\varepsilon \mathrm{r}=38.3)\end{array}$} & E kin & 724.8321 & 729.6968 & 734.5614 & 739.4261 & 744.2907 & 749.1554 & 754.02 & 758.8847 & 763.7493 & 768.6139 \\
\hline & E pot & 67352.25 & 50109.04 & 39000.96 & 31635.91 & 26596.85 & 22678.25 & 19522.2 & 17139.19 & 15221.89 & 13700.51 \\
\hline & E tot & 68077.08 & 50838.73 & 39735.52 & 32375.33 & 27340.93 & 23427.44 & 20276.22 & 17898.07 & 15985.64 & 14469.13 \\
\hline
\end{tabular}

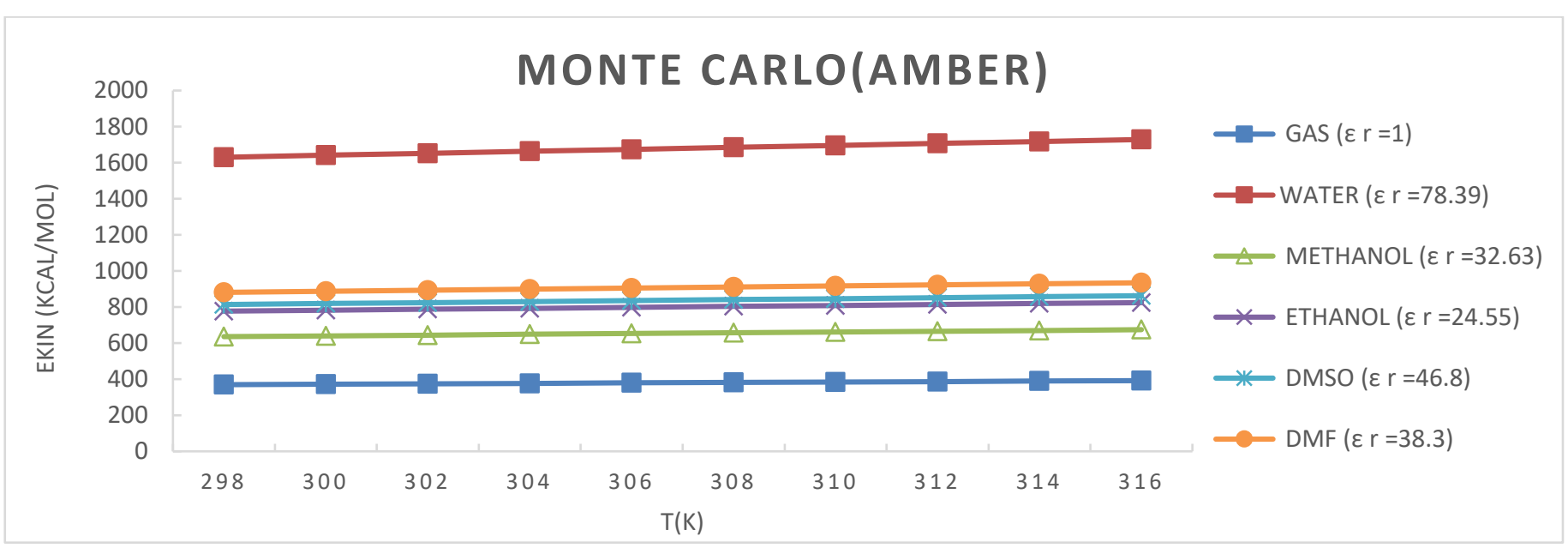

Figure 12. EKin changes (kcal.mol) calculated versus temperature at different dielectric constants through Monte Carlo simulation in the Amber force field for L-FMTX with BNNTs.

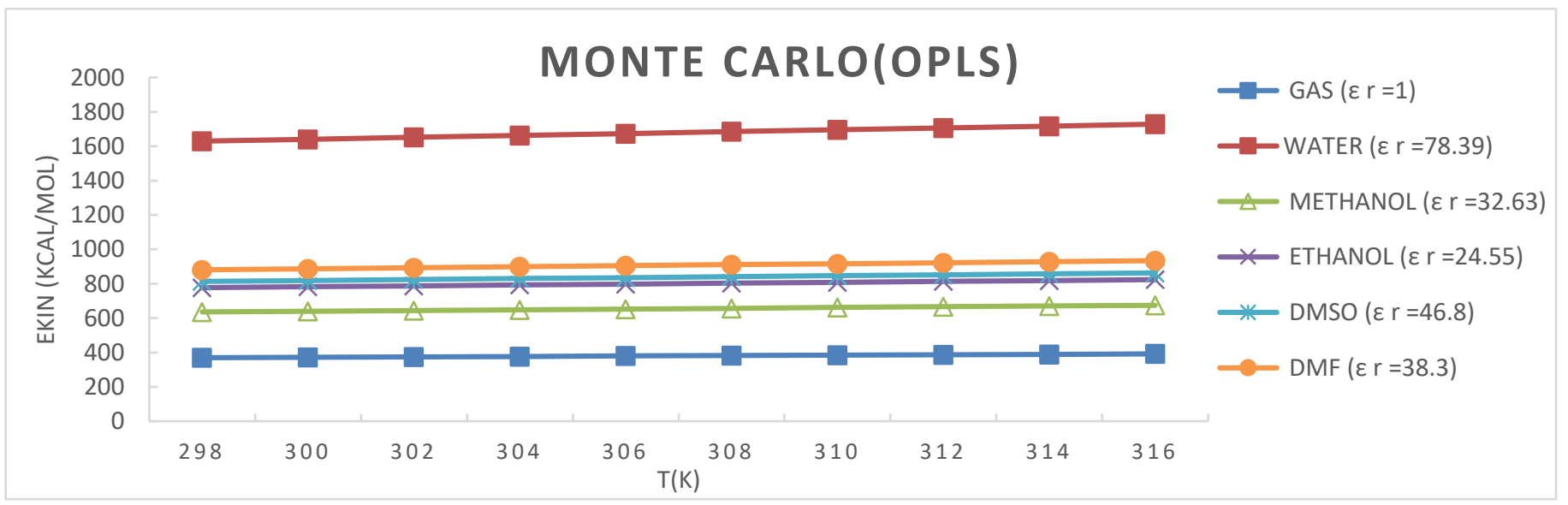

Figure 13. EKin changes (kcal.mol) calculated versus temperature at different dielectric constants through Monte Carlo simulation in the OPLS force field for L-FMTX with BNNTs. 


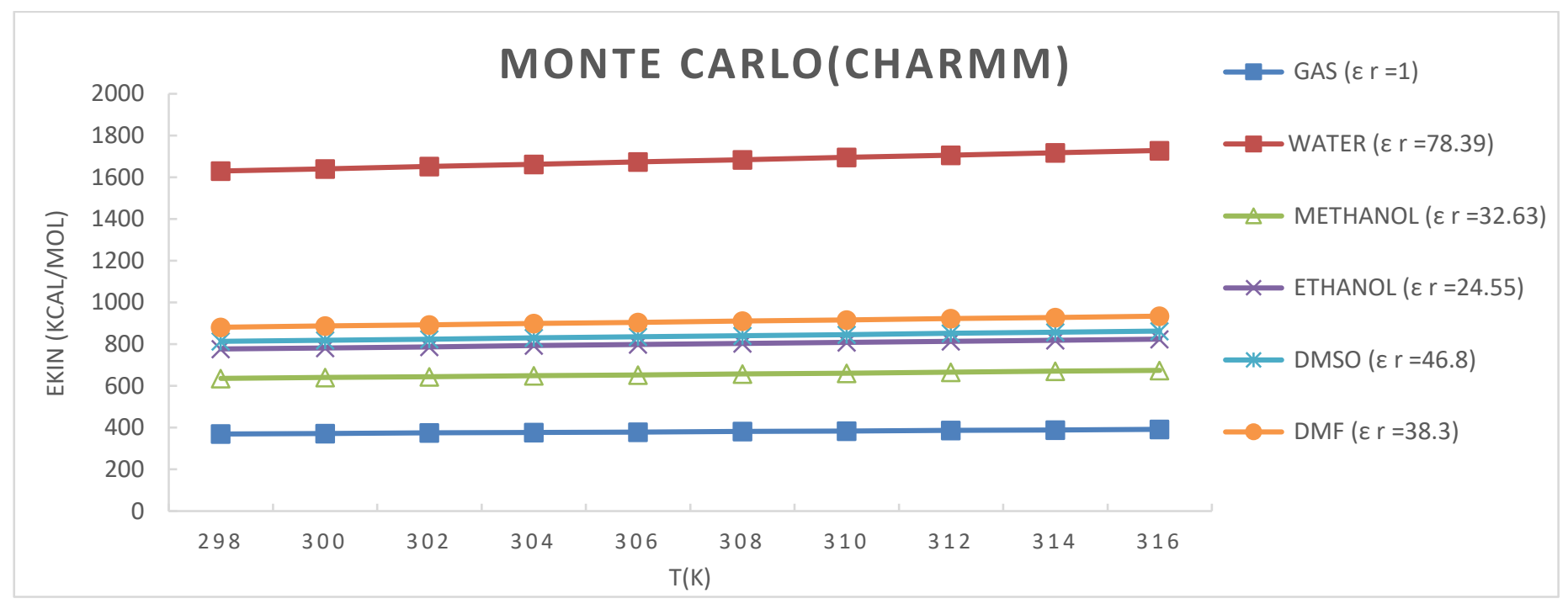

Figure 14. EKin changes (kcal.mol) calculated versus temperature at different dielectric constants through Monte Carlo simulation in the CHARMM force field for L-FMTX with BNNTs.

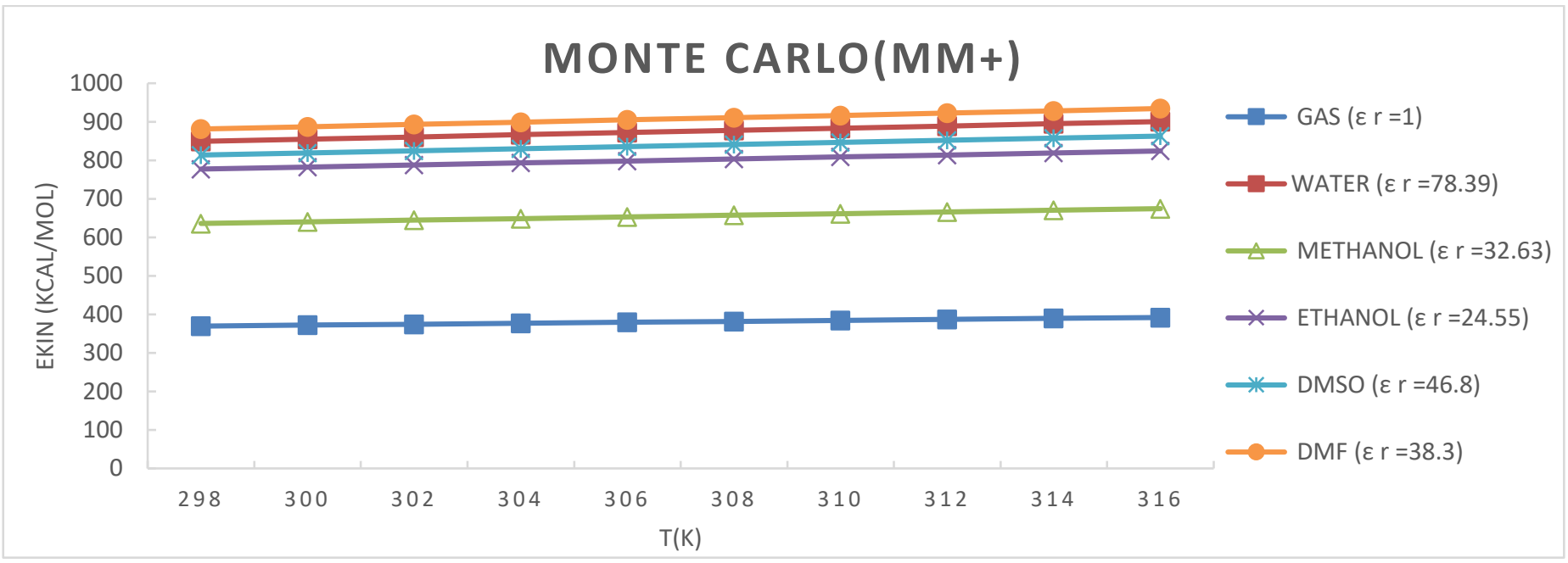

Figure 15. EKin changes (kcal.mol) calculated versus temperature at different dielectric constants through Monte Carlo simulation in the MM+ force field for L-FMTX with BNNTs.

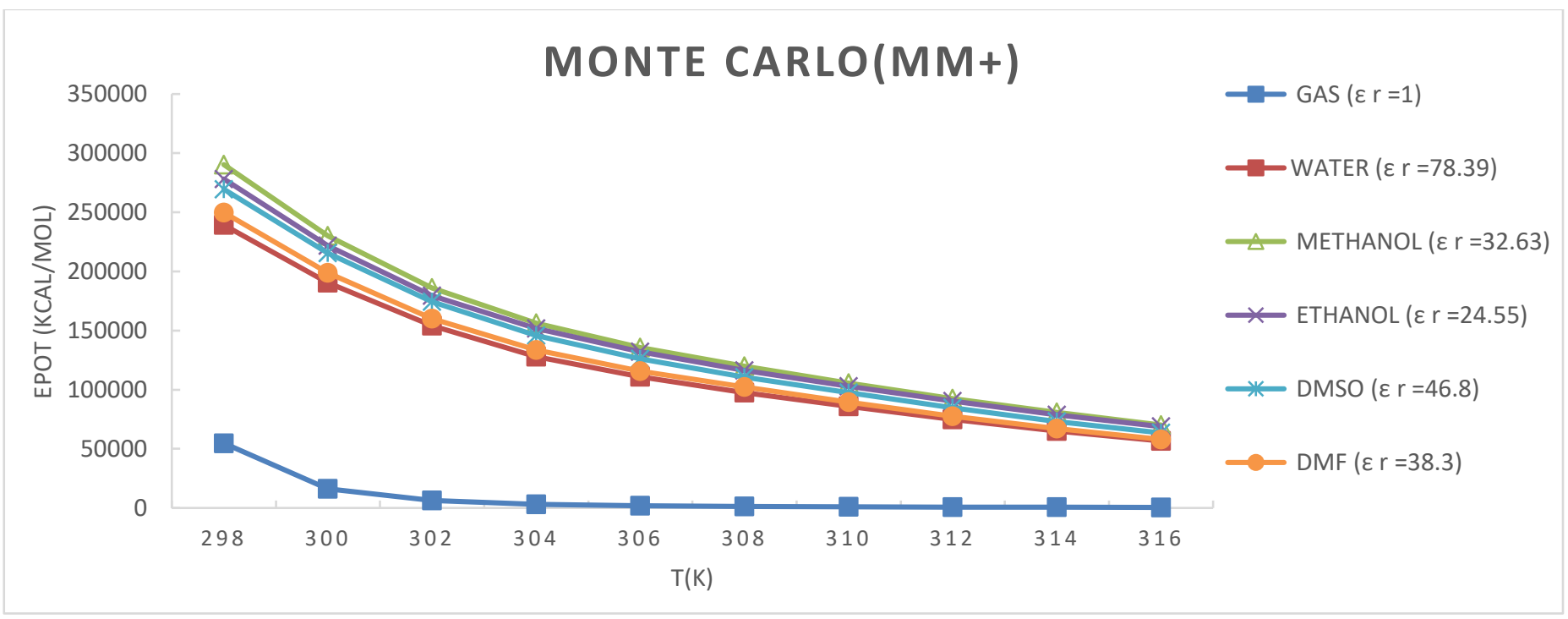

Figure 16. EPot changes (kcal.mol) calculated versus temperature at different dielectric constants through Monte Carlo simulation in the MM+ force field for L-FMTX with BNNTs. 


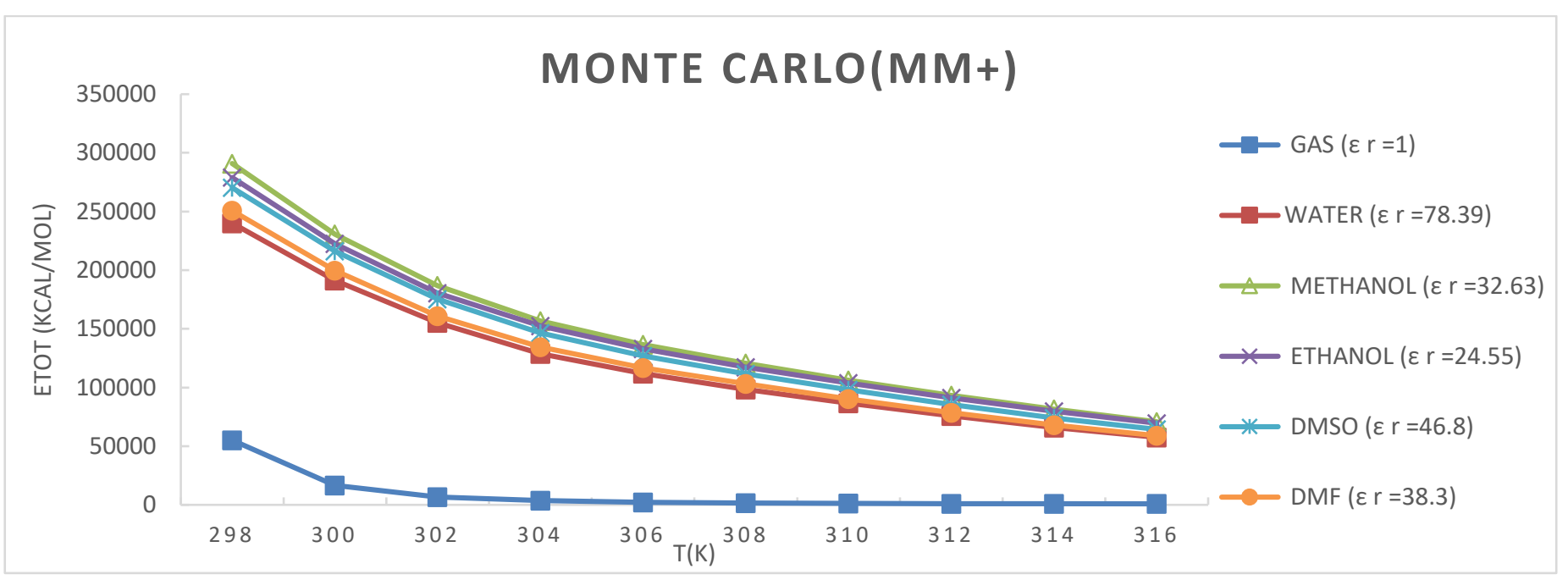

Figure 17. ETot changes (kcal.mol) calculated versus temperature at different dielectric constants through Monte Carlo simulation in the MM+ force field for L-FMTX with BNNTs.

\section{CONCLUSION}

In this study, the interaction of two effective derivatives of the anticancer drug methotrexate with Single-wall carbon nanotubes (SWNTs) and Boron nitride nanotubes (BNNTs) in the gas phase has been investigated using the DFT calculations. Through the DFT method, we studied the effects of different solvents on the interaction of methotrexate derivatives with SWNTs and BNNTs within the Onsager self-consistent reaction field (SCRF) model; we also studied the effects of temperature on the stability of the interaction between compounds in various solvents. We resorted to theoretical calculations for investigating Total Density of States (DOS), Frontier Molecular Orbitals (FMOs) and thermodynamic parameters of the title compounds. The molecular properties of the structures such as ionization potential (I), electron affinity (A), chemical hardness $(\eta)$, electronic chemical potential $(\mu)$ and electrophilicity $(\omega)$ were analysed. The data showed that the L-MTX and carbon nanotube combination had more stability. This was further confirmed by the amounts of HOMO and LUMO energies and Gibbs free energy. However, the results pertaining to LFMTX differed substantially with those related to L-MTX.

In addition, the data pointed to the fact that the L-FMTX and boron-nitride nanotube combination had more stability, which was also confirmed by the amounts of HOMO and LUMO energies. The two structures differed in the $\mathrm{H}$ and $\mathrm{F}$ constituents. High electronegativity of $\mathrm{F}$ compared to $\mathrm{H}$ resulted in the stability of both L-MTX and carbon nanotube combination, and L-FMTX and boron-nitride nanotube combination.

The effect of different solvents and temperatures on the L-MTX and L-FMTX (with SWNTs \& BNNTs) was studies through quantum mechanics calculations and molecular mechanic simulation. Differences in force fields were illustrated by comparing the energies calculated using AMBER, OPLS, CHARM (Bio+) and $\mathrm{MM}+$ force fields. The methanol solvent displayed the lowest amount of energy and proved to be the most stable solvent for the simulation, when L-MTX connected to SWNTs was simulated in water, DMSO, methanol, ethanol, $\mathrm{CH} 2 \mathrm{Cl} 2$ and DMF solvents. Similar results have been reported for OPLS and CHARMM force fields. However, the calculations related to the MM+ force field yielded a notable result. In the MM+ field, water was the most stable and the most suitable among the aforementioned solvents for simulation, since it had the lowest amount of energy. This was certainly positively related to the dielectric constant of the solvents. Water had the highest dielectric constant; therefore, it was considered to be the most suitable solvent for L-MTX connected to SWNTs.

It is noteworthy that the results for L-FMTX connected to BNNTs were highly consistent with those related to L-MTX connected to SWNTs; in the force fields Amber, OPLS and CHARMM, methanol was the most stable solvent and in the MM+ field, water was the most stable solvent. Finally, we found that the MM+, which is an exclusive force field for calculations related to macromolecules had the lowest amount of energy and featured the most stable form of connection for Methotrexate derivatives connected to SWNTs and BNNTs. Notably, in some solvents and at certain temperatures, the CHARMM force field demonstrated a similar behaviour and put our compound in a stable situation. However, since electrostatic reactions are calculated through bipolar junctions by using point charges in the MM+ field, the field managed to simulate our desired system in the most optimal way. Therefore, the MM+ force field was chosen as the most efficient field.

It should further be noted that the results of Quantum Mechanics calculations are also consistent with the current findings; SWNTs are more suitable carriers for L-MTX and BNNTs are more suitable carriers for L-FMTX. The results of Monte Carlo, Molecular Mechanics and Quantum Mechanics calculations are thus justified.

Delivering anti-cancer drugs through SWNTs and BNNTs is a considerable breakthrough in the field of nanotechnology. Conventional management of cancer with chemotherapeutic agents can have adverse effects on healthy tissues. Thus, development of CNTs -based efficient drug delivery systems is imperative for delivering anti-cancer drugs. Even though nano-technology is fairly developed, it is still far from clinical applications due to several challenges. However, SWCNTs- and BNNTs-based drug delivery systems are promising approaches for delivering anti-cancer drugs to targeted organs or tissues. The observations and results of this review paper indicated that SWNTs- and BNNTsbased drug delivery systems might be highly effective and able to provide adequate scientific data for clinical support.

\section{ACKNOWLEDGEMENTS}

The authors are thankful to the Ahvaz Branch of Islamic Azad University and the Zanjan Branch of Islamic Azad University for partial support of this work.

\section{REFERENCES}

1. X. Li, Y. Peng, X. Qu, Nucleic Acids Research. 34, 3670, (2006)

2. C. Klumpp, K. Kostarelos, M. Prato, A. Bianco, Biochimica et Biophysica Acta (BBA) - Biomembranes. 1758, 404, (2006)

3. N. Sinha, J. T. W. Yeow, IEEE transitions on nanobioscience. 4, 180, (2005)

4. N. Nishiyama, K. Kataoka, Pharmacology \& therapeutics. 112, 630, (2006)

5. H. Mizusako, T. Tagami, K. Hattori, T. Ozeki, Journal of pharmaceutical sciences. 104, 2934 (2015)

6. Q. He, J, Shi, Jurnal of Materials Chemistry. 21, 5845, (2011)

7. G. Kwon, S. Suwa, M. Yokoyama, T. Okano, Y. Sakurai, K. Kataoka, Journal of Controlled Release. 29, 17, (1994)

8. A. Mahmud, X. B. Xiong, H. M. Aliabadi, A. Lavasanifar, Journal of Drug Targeting. 15, 553 (2007)

9. S. Khatri, N. G. Das, S. K. Das, Journal of pharmacy \& bioallied sciences. 6, 297, (2014)

10. J. E. Kim, J. Y. Shin, M. H. Cho, Archives of Toxicology. 86, 685, (2012)

11. C. Sun, J. S. Lee, M. Zhang, Advanced drug delivery reviews. 60, 1252 , (2008) 
12. R. Arenal, A. Lopez-Bezanilla, Computational Molecular Science. 5, 299, (2015)

13. G. Ciofani, Expert opinion on drug delivery. 7, 889, (2010)

14. J. H. Kim, T.V. Pham, J. H. Hwang, C. S. Kim, M. J. Kim, Nano convergence. 5, 1, (2018)

15. J. Iqbal, K. Ayub, Journal of Alloys and Compounds. 687, 976, (2016)

16. D. Golberg,X. D. Bai, M. Mitome,C. C. Tang, C. Y. Zhi, Y. Bando, Acta materialia. 55, 1293, (2007)

17. D. Lahiri, F. Rouzaud, T. Richard, A. K. Keshri, S. R. Bakshi, L. Kos, A. Agarwal, Acta biomaterialia. 6, 3524, (2010)

18. W. An, C. H. Turner, the Journal of Physical Chemistry Letters. 1, 2269, (2010)

19. C. W. Chang, W. Q. Han, A. Zettl, Journal of Vacuum Science \& Technology B: Microelectronics and Nanometer Structures Processing, Measurement, and Phenomena. 23, 1883, (2005)

20. T. He, T. Li, A. Huang, Z. Tang, X. Guan, Physica E: Low-dimensional Systems and Nanostructures. 107, 182, (2019)

21. G. Ciofani, S. Danti, G. G. Genchi, B. Mazzolai, V. Mattoli, biocompatibility and potential spill-over in nanomedicine. Small. 9, 1672, (2013)

22. Z. Khatti, S. M. Hashemianzadeh, European Journal of Pharmaceutical Sciences. 88, 291, (2016)

23. F. Azarakhshi, M. Sheikhi, S. Shahab, M. Khaleghian, K. Sirotsina, H. Yurlevich, D. Novik, Chemical Papers. 75, 1521, (2021)

24.Z. Mahdavifar, R. Moridzadeh, Journal of Inclusion Phenomena and Macrocyclic Chemistry. 79, 443, (2014)

25. P. N. Samanta, K. K. Das, RSC advances. 6, 92547, (2016)

26. B. N. Cronstein, Arthritis \& Rheumatism. 39, 1951, (1996)

27. M. Wojtoniszak, K. Urbas, M. Perużyńska, M. Kurzawski, M. Droździk, E. Mijowska, Chemical Physics Letters. 568, 151, (2013)

28. H. Matsuoka, N. Ohi, M. Mihara,H. Suzuki, K. Miyamoto, N. Maruyama, T. Kuroki, Journal of medicinal chemistry. 40, 105, (1997)

29. T. M. Goszczyński, B, Filip-Psurska, K. Kempińska, J. Wietrzyk, J. Boratyński, Pharmacology research \& perspectives. 2, e00047, (2014)

30. R. Segal, M. Yaron, B. Tartakovsky, arthritis and rheumatism. 20, 190, (1990)

31. J. A. Moura, C. J. Valduga, E. R. Tavares, I. F. Kretzer, D. A. Maria, R. C. Maranhão, journal of nanomedicine. 6, 2285, (2011)

32. S. Moura, J. Noro, P. Cerqueira, C. Silva, A. Cavaco-Paulo, A. Loureiro, International journal of pharmaceutics. 575, 118924, (2020)

33. R. J. Kempton, A. M. Black, G. M. Anstead, A. A. Kumar, d. T. Blankenship, J. H. Freisheim, Journal of medicinal chemistry. 25, 475-(1982)

34. A. Nemat, I. N. Khan, S. Kalsoom, S. A. Malik, S. Ayub, F. Adnan, M. A. Kamal, M. Iqbal, Journal of Biomolecular Structure and Dynamics. 38, 1, (2020)

35. Y. Kokuryo, K. Kawata, T. Nakatani, A. Kugimiya, Y. Tamura, K. Kawada, M. Ohtani, Journal of medicinal chemistry. 40, 3280, (1997)

36. N. Uddin, S. Ahmed, A. M. Khan, M. Mazharol Hoque, M. A. Halim, Journal of Biomolecular Structure and Dynamics. 38, 901, (2020).

37. S. Ekins, J. Mestres, B. Testa, British Journal of Pharmacology. 152, 21, (2007)

38. L. Fraenkel, W. B. Nowell, G. Michel, C. Wiedmeyer. Annals of the Rheumatic Diseases. 77, 678, (2018)
39. A. L. Mathews, A. Coleska, P. B. Burns, K. C. Chung, Arthritis Care \& Research. 68, 318, (2016)

40. P. Workman, Cold Spring Harbor Symposia on Quantitative Biology. 70 , 499, (2005)

41. I. Collins, P. Workman, Nature Chemical Biology. 2, 689, (2006)

42. A. D. MacKerell, D. Bashford, M. Bellott, et al, J. Phys. Chem. B. 102, 3586, (1998)

43. A. D. MacKerell Jr, M. Feig, Journal of Computational Chemistry. 25, 1400, (2004)

44. W. L. Jorgensen, D. S. Maxwell, J. Tirado-Rives, J. Am. Chem. Soc. 118, $11225,(1996)$

45. V. Hornak, R. Abel, A Okur, B. Strockbine, A. Roitberg, C. Simmerling, Proteins. 65, 712, (2006)

46. M. J. Frisch, G. W. Trucks, H. B. Schlegel, et al, Gaussian 09 revision A02, Gaussian, Inc., Wallingford CT, (2009)

47. A. Frisch, A. B. Nielson, A. J. Holder, 2000, GAUSSVIEW User Manual, Gaussian Inc.; Pittsburgh, PA.

48. A. D.J. Becke, Chem. Phys. 98, 5648, (1993)

49. J. Tomasi, B. Mennucci, R. Cammi, Chem. Rev. 105, 2999, (2005)

50. S. Shahab, M. Sheikhi, L. Filippovich, E. Dikusar, H. Yahyaei, R. Kumar, M Khaleghian, J. Mol. Struct. 1157, 536, (2018)

51. H. Yahyaei, S. Sharifi, S. Shahab, M. Sheikhi. M. Ahmadianarog, Letters in Organic Chemistry. 18, 115, (2021)

52. S. Shahab, M. Sheikhi, L. Filippovich, R. Kumar, E. Dikusar, H. Yahyaei, M. Khaleghian, Journal of Molecular Structure. 1148, 134, (2017)

53. [53] A. Szczepanska, J. L. Espartero, A. Moreno-Vargas, A. T. Carmona, I. Robina, S. Remmert, C. Parish, J. Org. Chem. 72, 6776, (2007)

54. N. Metropolis, A. W. Rosenbluth, M. N. Rosenbluth, A. H. Teller, E. Teller, J. Chem. Phys. 21, 1087, (2004)

55. HyperChem 7.0. (2001) Hypecube Inc., FL. Gainesville,

56. H. Yahyaei, M. Monajjemi, H. Aghaie, K. Zare, Journal of Computational and Theoretical Nanoscience. 10, 2332, (2013)

57. M. Kastner, Communications in Nonlinear Science and Numerical Simulation. 15, 1589, (2010)

58. W. K. Hastings, Biometrika. 57, 97, (1970)

59. J. S. Liu, F. Liang, W. H. Wong, Journal of the American Statistical Association. 95, 121, (2000)

60. J. A. Champion, S. Mitragotri, Natl Aead Sei. 103, 4930, (2006)

61. SL. Kinnings, N. Liu, N. Buchmeier, P. J. Tonge, L. Xie, P. E. Bourne, PLOS Computational Biology. 5, e1000423, (2009)

62. I. Collins, P. Workman, Nature Chemical Biology. 2, 689, (2006)

63. N. Scheinfeld, Dermatology online journal. 12, 15, (2006)

64. BN. Cronstein, Pharmacol Rev. 57, 163, (2005)

65. M. R. Sawaya, J. Kraut, Biochemistry, 36,586, (1997)

66. L. M. Meyer, F. R. Miller, M. J. Rowen, G. Bock, J. Rutzky, Acta Haematologica. 4, 1571, (1950)

67. S. Majedi, L. Sreerama, E. Vessally, F. Behmagham, J. Chem. Lett. 1, 25 (2020).

68. N. Shajari, H. Yahyaei, physical chemistry research, 4, 705, (2020)

69. H. Yahyaei, S. Shahab, M. Sheikhi, et al, Spectrochimica Acta Part A: Molecular and Biomolecular Spectroscopy, 192, 343, (2018) 